\title{
ARTICLE
}

\section{Hace1 controls ROS generation of vertebrate Rac1-dependent NADPH oxidase complexes}

Mads Daugaard ${ }^{1,2}$, Roberto Nitsch ${ }^{3}$, Babak Razaghi ${ }^{4}$, Lindsay McDonald ${ }^{4}$, Ameer Jarrar ${ }^{4}$, Stéphanie Torrino ${ }^{5}$, Sonia Castillo-Lluva ${ }^{6}$, Barak Rotblat ${ }^{1}$, Liheng Li ${ }^{1}$, Angeliki Malliri ${ }^{6}$, Emmanuel Lemichez ${ }^{5}$, Amel Mettouchi ${ }^{5}$, Jason N. Berman ${ }^{4}$, Josef M. Penninger ${ }^{3}$ \& Poul H. Sorensen ${ }^{1,2}$

The Hace1-HECT E3 ligase is a tumor suppressor that ubiquitylates the activated GTP-bound form of the Rho family GTPase Rac1, leading to Rac1 proteasomal degradation. Here we show that, in vertebrates, Hace1 targets Rac1 for degradation when Rac1 is localized to the nicotinamide adenine dinucleotide phosphate (NADPH) oxidase holoenzyme. This event blocks de novo reactive oxygen species generation by Rac1-dependent NADPH oxidases, and thereby confers cellular protection from reactive oxygen species-induced DNA damage and cyclin D1-driven hyper-proliferation. Genetic inactivation of Hace1 in mice or zebrafish, as well as Hace1 loss in human tumor cell lines or primary murine or human tumors, leads to chronic NADPH oxidase-dependent reactive oxygen species elevation, DNA damage responses and enhanced cyclin D1 expression. Our data reveal a conserved ubiquitin-dependent molecular mechanism that controls the activity of Rac1-dependent NADPH oxidase complexes, and thus constitutes the first known example of a tumor suppressor protein that directly regulates reactive oxygen species production in vertebrates.

\footnotetext{
${ }^{1}$ Department of Molecular Oncology, British Columbia Cancer Research Centre, Vancouver, British Columbia V5Z 1L3, Canada. ${ }^{2}$ Department of Pathology and Laboratory Medicine, University of British Columbia, Vancouver, British Columbia V6T 1Z4, Canada. ${ }^{3}$ IMBA, Institute of Molecular Biotechnology of the Austrian Academy of Sciences, Dr Bohrgasse 3, Vienna 1030 Austria. ${ }^{4}$ Department of Pediatrics, Dalhousie University and IWK Health Centre, Halifax, Nova Scotia B3K 6R8, Canada. ${ }^{5}$ Equipe labellisée Ligue Contre Le Cancer, U1065, Centre Méditerranéen de Médecine Moléculaire, C3M, Université de NiceSophia-Antipolis, 806204 Nice, France. ${ }^{6}$ Cell Signalling Group, Cancer Research UK Paterson Institute for Cancer Research, The University of Manchester, Manchester, M20 4BX, UK. Correspondence and requests for materials should be addressed to P.H.S. (email: phbsorensen@gmail.com).
} 
ACE1 (HECT domain and Ankyrin repeat Containing E3 ubiquitin-protein ligase 1), a tumor suppressor gene originally cloned from inactivating chromosome 6q21 breakpoints in human Wilms' tumor, is found exclusively in vertebrates and is widely expressed in human tissues ${ }^{1}$. It is epigenetically inactivated in human Wilms' tumors and several other tumor types ${ }^{1-6}$. In a recent study, loss of Hacel expression is associated with neuroblastoma progression and predicts poor overall patient survival $^{2}$. These observations are consistent with HACE1 functioning as a chromosome 6q21 tumor suppressor gene. Indeed, Hace $1-/-$ mice develop late-onset (18-24 months) spontaneous tumors across all three germ layers ${ }^{6}$. Tumor development is dramatically accelerated by DNA damage inducing agents such as low-dose ionizing radiation (IR) or the DNA alkylating agent, urethane ${ }^{6}$, but the basis for this hypersensitivity is not understood. Hacel reduces in vitro and in vivo cell cycle progression of diverse tumor cell lines such as KRAS-transformed NIH3T3 fibroblasts or human Ewing sarcoma SKNEP1 cells ${ }^{6}$. Re-expression of Hace1 in these cells blocks in vitro and in vivo proliferation, particularly after cell stress such as IR, nutrient deprivation or contact inhibition. This correlates with marked repression of cyclin D1 protein levels ${ }^{6}$, a key G1-S progression factor ${ }^{7}$. After serum deprivation or IR, Hace1 blocks cell cycle re-entry in an E3 ligase-dependent manner through failure of cells to re-express cyclin D1, while other cyclins are unaffected. This occurs in a ligase-dependent manner, although Hacel does not itself target cyclin D1 for ubiquitylation ${ }^{6}$. D-type cyclins have well-established roles in oncogenesis, and overexpression of cyclin D1 is reported in diverse human malignancies ${ }^{8}$. Knockdown of endogenous Hace1 in HEK293 cells (which express high endogenous Hacel levels) by RNA interference stabilizes cyclin D1 protein levels, and ectopic expression of Hace1 blocks cyclin D1 expression, as well as in vitro and in vivo cell cycle progression of Hace1-deficient human tumor cell lines ${ }^{6}$.

Currently, the only known Hace1 E3 ligase substrate is the GTP-bound form of the Rho GTPase, Racl. The latter is bound and ubiquitylated by Hacel at lysine-147 (Lys-147), leading to Rac1 proteasomal degradation and reduced cell motility in response to cytotoxic necrotizing factor-1 (CNF1) or hepatocyte growth factor ${ }^{9,10}$. Rac1 localizes to various cellular compartments and regulates multiple processes including cell motility ${ }^{11}$, protein translation $^{12}$, stress signaling ${ }^{13}$, proliferation ${ }^{14}$ and reactive oxygen species (ROS) generation ${ }^{15,16}$. How Rac1 activity orchestrates such diverse functions is poorly understood, but evidence suggests that subcellular localization of activated Rac1 is a critical factor ${ }^{15,17,18}$. Hacel co-localizes with a small fraction of total Rac1 in cells, corresponding to the active (GTP-bound) form of the protein, at any given time ${ }^{9}$. Moreover, a second class of E3 ligases, X-linked and cellular inhibitors of apoptosis IAP1 (XIAP and c-IAP1, respectively) directly bind Rac1 in a nucleotideindependent manner and promote Lys-147 polyubiquitylation and proteasomal degradation ${ }^{19}$. This suggests that different E3 ligases might target Rac1 at distinct subcellular sites.

In the current study, we sought to uncover why Hacel deficiency confers in vivo hypersensitivity to IR and urethane, and whether this is linked to Racl targeting by Hacel. Besides the mitochondria, the main sources of cellular ROS are plasma membrane and endosomal nicotinamide adenine dinucleotide phosphate (NADPH) oxidase complexes ${ }^{20}$. These are the only known mammalian enzymes exclusively dedicated to the production of superoxide, which is rapidly converted to $\mathrm{H}_{2} \mathrm{O}_{2}$ in the cell ${ }^{21}$. The NADPH oxidase holoenzyme consists of transmembrane catalytic cell type specific Nox subunits 1-5 (predominantly Nox1 and Nox 2 in epithelial and mesenchymal cells), the transmembrane p22phox protein, and the cofactors p47phox, p67phox, NOXA1 and NOXO1 (ref. 21). Rac1 GTPase binds NOXA1 within the complex and this is required for activation of Nox1, 2 and 3-containing NADPH oxidase enzymes $^{16,21}$. Accordingly, we hypothesized that increased ROS in Hace1-deficient cells might derive from hyperactivation of NADPH oxidase due to Racl stabilization.

Here we show that Hacel binds and ubiquitylates Racl when the latter is associated with NOXA1 at the NADPH oxidase complex, thus blocking ROS generation by the complex. Moreover, loss of Hacel in human tumors and cell lines, mice and zebrafish results in ROS-induced DNA damage and cyclin D1 induction, each of which depends on Racl and NADPH oxidase activity. Taken together, our results reveal Hacel as the first known negative regulator of NADPH oxidase, and highlight the role of deregulated NADPH oxidase activity in ROS-induced DNA damage and cell cycle progression.

\section{Results}

Hacel deficiency leads to chronic oxidative stress. IR and urethane, which both dramatically accelerate tumor formation in Hace $1^{-/-}$mice $^{6}$, are each known to induce high levels of ROS, particularly $\mathrm{H}_{2} \mathrm{O}_{2}$ (refs 22,23). We therefore speculated that Hace1 might itself regulate cellular ROS levels. Indeed, measurement of ROS levels in control and Hace 1-/ - MEFs with $2^{\prime}, 7^{\prime}$ dichlorodihydrofluorescein diacetate (DCFDA) ${ }^{24}$ showed a marked baseline elevation of ROS in Hace1-/MEFs, which could be almost completely reversed by reexpression of wild-type (wt) Hace1 (Fig. 1a). Increased ROS was also detected in Hace $1^{-/-}$MEFs with a second sensor, dihydroethidium (DHE), which is converted to the red fluorescent compound oxyethidium in vitro and in vivo in the presence of $\operatorname{ROS}^{25}$ (Fig. 1b and Supplementary Fig. S1a). Staining with MitoSoxRed, which specifically measures mitochondrial ROS, was largely unchanged between Hace ${ }^{+/+}$and Hace $1^{-/-}$ cells. We then used nitrotetrazolium blue (NTB) to visualize ROS in Hace $1-I-$ and $+/+$ MEFs, which clearly demonstrated formazan dots along the cell membranes and also in punctate dots within the cytoplasm in Hace $1-/$ - cells (Supplementary Fig. S1b). These data are consistent with Hacel deficiency resulting in massive accumulation of ROS from membraneassociated NADPH oxidase complexes, but it is possible that under chronic ROS accumulation additional ROS sources are activated, such as mitochondria. To confirm a role for Hacel in regulating ROS levels, we knocked down Hacel expression in human embryonic kidney (HEK) 293 cells using two nonoverlapping short interfering RNAs (siRNAs) (Supplementary Fig. S1c). Total ROS levels increased by 4 - to 6 -fold in Hace1 knockdown (kd) cells compared with controls (Fig. 1d). Moreover, all organs isolated from Hace $1^{-/-}$mice showed dramatic increases in ROS compared with those of littermate controls (Fig. 1e). Therefore, Hacel deficiency in mammalian cells leads to chronic high ROS levels in vitro and in vivo. To determine whether Hacel regulation of cellular ROS is conserved, we identified the hace 1 homologue in zebrafish, which displays $74.7 \%$ DNA sequence homology and $88.9 \%$ protein identity with human HACE1 (Supplementary Fig. S2a,b). A hace1 morpholino was designed to knock down hacel expression (Supplementary Fig. S2c,d), and zebrafish embryos were incubated over 1-7 days with the $\mathrm{H}_{2} \mathrm{O}_{2}$-specific probe, pentafluorobenzenesulphonyl fluorescein, which is converted to a fluorescent form by $\mathrm{H}_{2} \mathrm{O}_{2}$ (ref. 26). A significant increase in ROS levels was observed in hace1 morpholinos compared with control morpholinos or uninjected embryos (Fig. 1f). This could be completely rescued by addition of the broad flavoenzyme inhibitor diphenylene iodonium (DPI) ${ }^{27}$ (Fig. 1f) or the panoxidase inhibitor, apocynin 
a

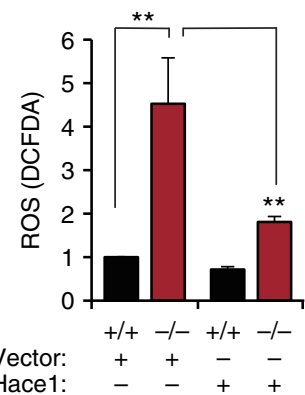

b

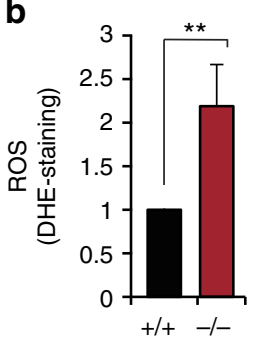

C

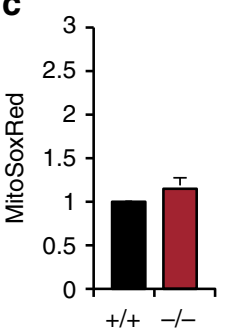

d

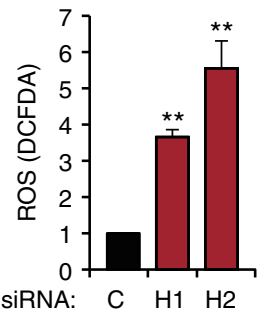

$\mathbf{e}$
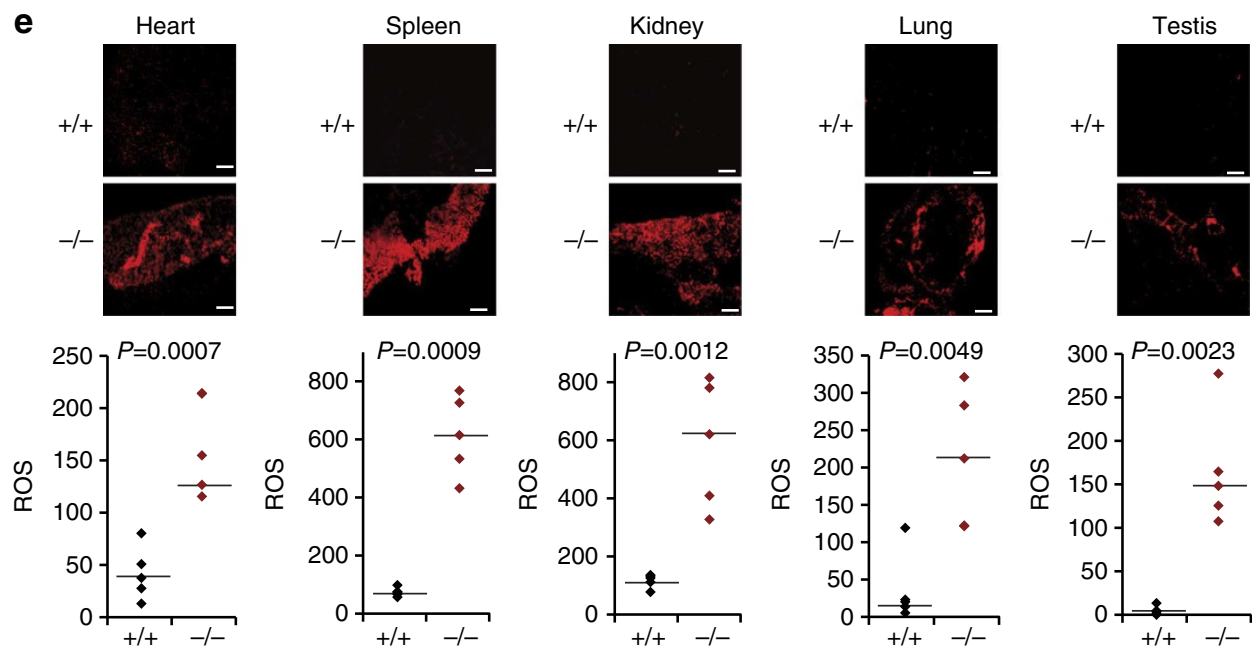

$\mathbf{f}$
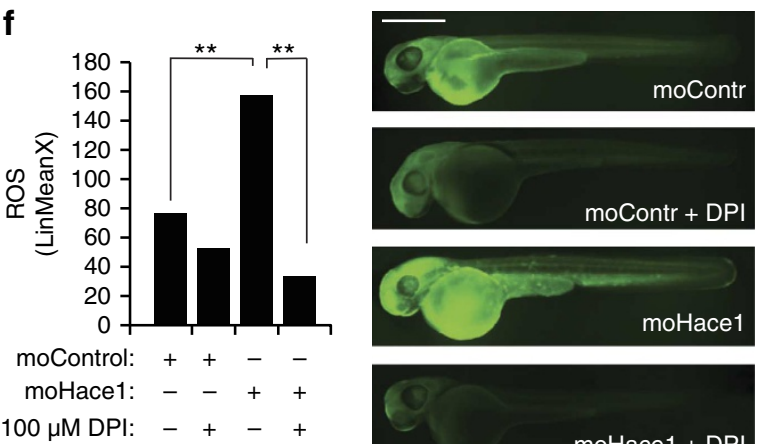

moHace1 + DPI

Figure 1 | Hace1 deficiency leads to elevated levels of ROS. (a) MEFs derived from wild-type $(+/+)$ or Hace1 knockout $(-/-)$ mice were transfected with the HA-vector $(\mathrm{V})$ or HA-Hace1 $(\mathrm{H}) 48 \mathrm{~h}$ and analyzed for ROS content by the DCFDA assay. (b) MEFs analogous to a were incubated with $10 \mu \mathrm{M}$ DHE for $30 \mathrm{~min}$, analyzed for oxyethidium staining by a 595-nm enabled fluorescence microscope and quantified using Image algorithm. (c) MEFs as in a were analyzed for mitochondrial derived ROS by the MitoSoxRed probe (Invitrogen). (d) HEK293 cells were transfected with control siRNA (C) or two siRNAs targeting Hace1 $(\mathrm{H} 1$ and $\mathrm{H} 2)$ for $72 \mathrm{~h}$ and analyzed as in $\mathbf{b}$. (e) Snap-frozen samples of the indicated tissues specimens from Hace1 wild-type $(+/+)$ and knockout $(-/-)$ mice were cut in $20 \mu \mathrm{m}$ sections using a cryostat at $-20^{\circ} \mathrm{C}$, incubated with $10 \mu \mathrm{M} \mathrm{DHE}$ for 30 min and analyzed as in $\mathbf{b}$. The $p$ values were generated using student's two-tailed $t$-test for equal variance. Scale bars, $20 \mu \mathrm{m}$. Horizontal lines in the graphs represent median values of quantification by ImageJ algorithm. (f) Representative images of $48 \mathrm{~h}$ post fertilization (hpf) casper zebrafish control ( $\mathrm{mC}$ ) and hace1 $(\mathrm{mH})$ morphants stained for ROS using pentafluorobenzenesulphonyl fluorescein. Both control and Hace1 morphants were left untreated or incubated with DPI as indicated, before being analyzed for ROS-induced fluorescence by FACS. For all panels when indicated, ${ }^{\star \star} P<0.01$ (Student's two-tailed $t$-test for equal variance); Error bars represent s.e.m. of at least three independent experiments. Scale bar, $0.5 \mathrm{~mm}$.

$(\mathrm{APO})^{28}$ (Supplementary Fig. S2e), both of which have welldocumented antioxidant activities. Together, these data demonstrate that Hacel deficiency results in massive accumulation of ROS in vertebrates.

Rac1 is required for ROS induction in Hace1-deficient cells. As increased ROS in Hacel-deficient cells is not mitochondrially derived, we speculated that Hacel might regulate NADPH oxidase complexes, another major source of cellular ROS. First, we analyzed ROS levels in Hace $1^{-/-}$MEFs after treatment with 2-Acethylphenothiazine (ML171), which specifically inhibits Nox1-containing NADPH oxidases $^{29}$, and two agents that block cellular ROS accumulation, $\mathrm{DPI}^{27}$ and $\mathrm{APO}^{28}$. All three inhibitors reduced ROS levels as effectively as $w t$ Hacel re-expression (Fig. 2a and Supplementary Fig. S3a). We then analyzed whether genetic loss of NADPH oxidase components influences ROS levels in Hace1-deficient cells. Indeed, siRNA knockdown of 
a

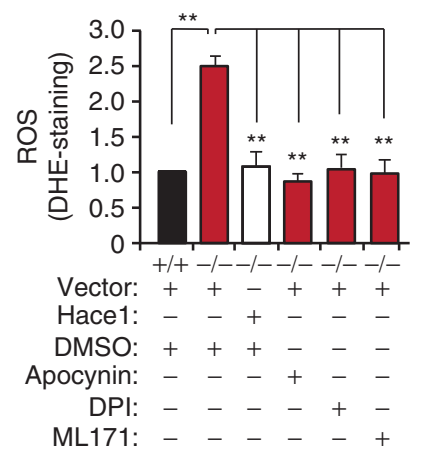

b

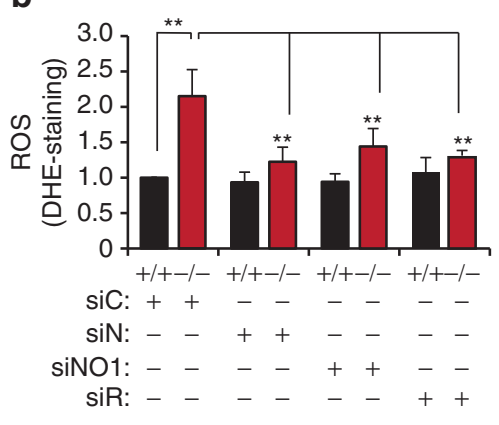

C

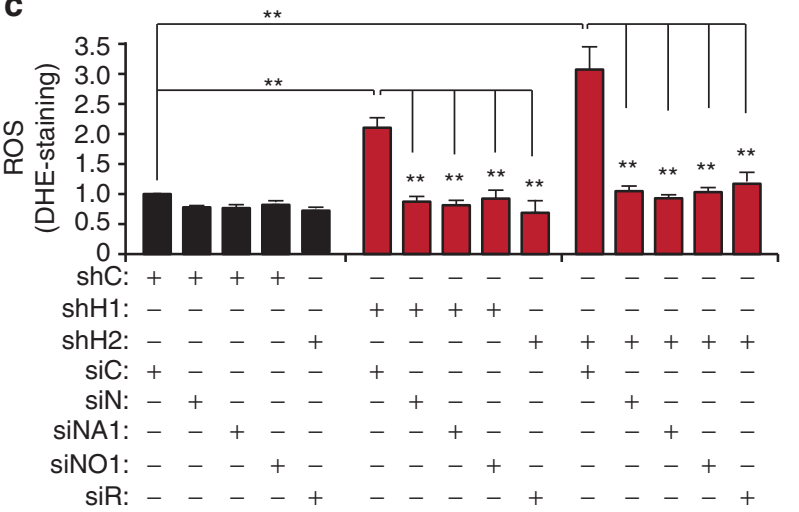

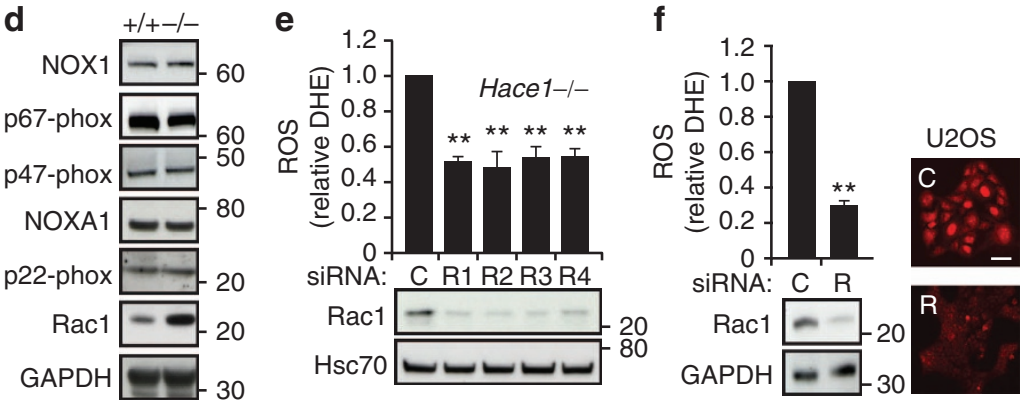

h

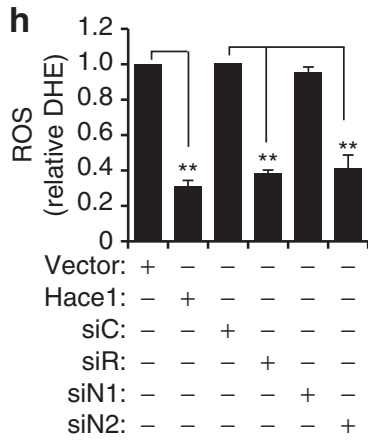

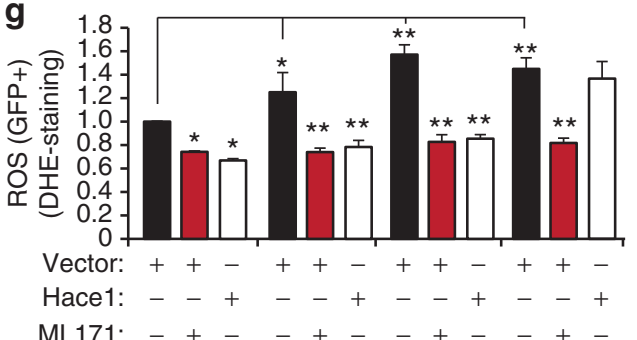

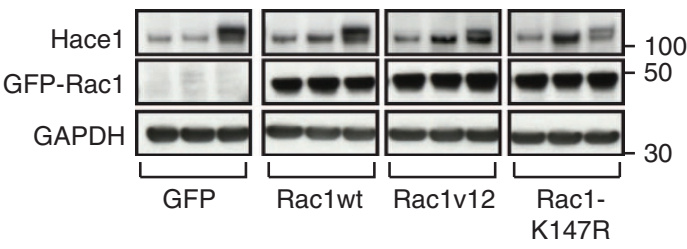

Figure 2 | Rac1 is required for increased ROS levels in Hace1-deficient cells. (a) MEFs derived from wild-type $(+/+)$ or Hace1 knockout $(-/-)$ mice transfected with HA-vector (Vector) or HA-Hace1 (Hace1) cDNA were left untreated (dimethylsulphoxide) or treated with $5 \mu \mathrm{M} \mathrm{ML171,5 \mu M} \mathrm{DPI} \mathrm{or}$ $400 \mu \mathrm{M}$ APO before incubated with DHE, analyzed for oxyethidium staining by a 595-nm enabled fluorescence microscope and quantified using Image algorithm. (b) MEFs analogous to a transfected with control (siC), Nox1 (siN), NOXO1 (siNO1) or Rac1 (siR) siRNAs were treated and analyzed as in a. (c) HEK293 cells expressing short hairpin RNA control (shC) or Hace1 (shH1 and shH2) shRNAs and transiently transfected with control (siC), Nox1 (siN), NOXA1 (siNA1), NOXO1 (siNO1) or Rac1 (siR) siRNAs were treated and analyzed as in a. (d) Protein lysates from MEFs derived as in a were analyzed by immunoblotting for the indicated proteins. (e) MEFs derived as in a transfected with control siRNA (C) or siRNAs targeting Rac1 (R1-4) were analyzed for ROS content as in a (graph column) and for expression of the indicated proteins by immunoblotting. (f) U2OS cells were transfected with control siRNA (C) or a siRNA targeting Rac1 (R) before analyzed for ROS content as in a (graph column and sample images) and for expression of the indicated proteins by immunoblotting. Scale bar, $10 \mu \mathrm{m}$. (g) U2OS cells transfected with combinations of HA-vector, HA-Hace1, GFP-vector, GFP-Rac1, GFP-Rac1-v12 and GFP-Rac1-K147R and treated with ML171 as indicated, were analyzed for ROS (upper panels) as in a. Protein lysates from the same cells were analyzed for expression of the indicated proteins by immunoblotting (lower). (h) SKOV3 cells were transfected with HA-vector (Vector) or HA-Hace1 (Hace1) cDNA for 48 h, or with control (siC), Nox1 (siN1), Nox2 (siN2) or Rac1 (siR) siRNAs were incubated with DHE and analyzed as in a. For all panels when indicated, ${ }^{\star} P<0.05$; ${ }^{\star} P<0.01$ (Student's two-tailed $t$-test for equal variance); Error bars represent s.e.m. of at least three independent experiments.

Nox1, NOXO1 or Rac1, all components of Nox1-containing NADPH oxidase ${ }^{21}$, in Hace $1^{-1-}$ MEFs, reversed the increase in ROS to the levels of $\mathrm{Hace}^{+/+}$cells (Fig. $2 \mathrm{~b}$ and Supplementary Fig. S3b). Consistent with this, ROS elevation by independent Hace1 siRNAs in human HEK293 cells (as in Fig. 1d) was reversed by transient siRNA-mediated co-knockdown of Nox1, NOXA1, NOXO1 or Rac1 (Fig. 2c and Supplementary Fig. S3c). Thus, Hacel inhibits the production of cellular ROS generated by Nox1-containing NADPH oxidase. Next, we analyzed protein levels of known NADPH oxidase subunits in $\mathrm{Hace1}^{-/-}$versus
Hace1 ${ }^{+/+}$MEFs. Both cell types displayed equivalent levels of each protein except Racl, which was markedly elevated in Hace $1^{-/}$MEFs (Fig. 2d). Indeed, Racl knockdown using independent siRNAs significantly reduced ROS in Hace $1^{-/-}$ MEFs (Fig. 2e), and in human U2OS osteosarcoma cells (Fig. 2f). This suggests that elevated ROS in Hace1-deficient cells originates from Rac1-dependent NADPH oxidases. GTP-loaded Rac1, the only known target of the Hacel E3 ligase, is essential for activation of Nox1, Nox2 and Nox3 containing NADPH oxidases $^{30}$. We therefore investigated whether the mechanism 
by which Hace1 regulates NADPH oxidase and cellular ROS levels relies on Rac1. GTP-bound Rac1 is ubiquitylated by Hacel at lysine (K)-147 (ref. 10). We therefore analyzed ROS levels in $\mathrm{U} 2 \mathrm{OS}$ cells after overexpression of $w t$ Rac1, a constitutively active Rac1-v12, or a Hace1-resistant Rac1-K147R mutant ${ }^{10}$. Both $w t$ Racl and the Rac1-v12 mutant significantly increased ROS levels, which could be reversed by either the ML171 NADPH oxidase inhibitor or Hacel overexpression (Fig. 2g). In contrast, ROS levels in cells expressing Hace1-resistant Rac1-K147R were not affected by Hacel overexpression, indicating that Hacel must directly target Rac1 to reduce ROS levels. We then tested Hace1 effects in SKOV3 ovarian carcinoma cells, which predominantly express Nox2 rather than Nox1 or Nox3 (Supplementary Fig. S3d and ref. 31). As shown in Fig. 2h, Hacel also strongly inhibited ROS generation in SKOV3 cells, and siRNAs to Nox2 but not to
Nox1 decreases ROS in SKOV3 cells to the same extent as observed with Hacel overexpression or Racl knockdown (Fig. $2 \mathrm{~h}$ and Supplementary Fig. S3e,f). Therefore, Hace1 regulates a second Rac1-dependent complex, namely Nox2-containing NADPH oxidase. Together, these data indicate that Hacel regulates multiple Rac1-dependent NADPH oxidases by targeting Racl for degradation.

Hace1 targets Rac1 bound to the NADPH oxidase complex. As Racl is an essential component of Nox1, 2 and 3-containing NADPH oxidase $^{32}$, we hypothesized that Hacel specifically targets for degradation the fraction of Racl that is localized and bound to the holoenzyme. We first confirmed that Rac1 is ubiquitylated by Hacel in our system by re-expressing Hacel in Hace1 - / - MEFs (Fig. 3a), consistent with previous findings ${ }^{9,10}$.

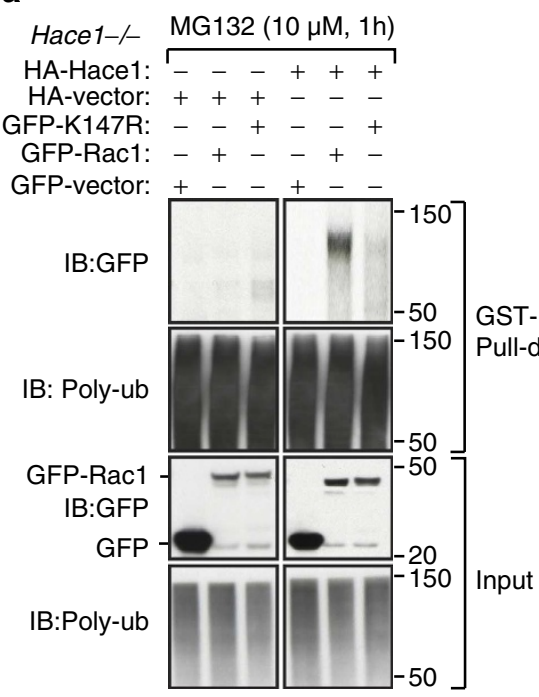

d
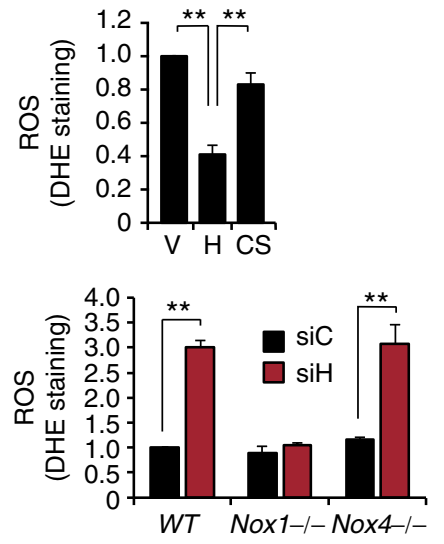

b

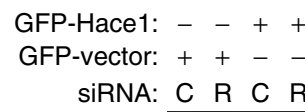

IB:GFP-Hace1

IB:Rac1

IB:NOXA1
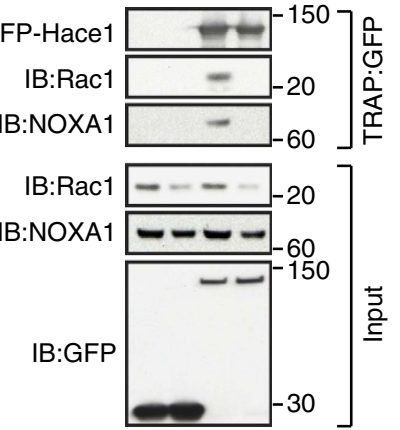

C

GST-Tube: --++++++

MG132: $++-\ldots+++$

HA-vector: +-+-+-+-

HA-Hace1: -+-+-+-+

SiRNA: C C C C C C NA1NA1

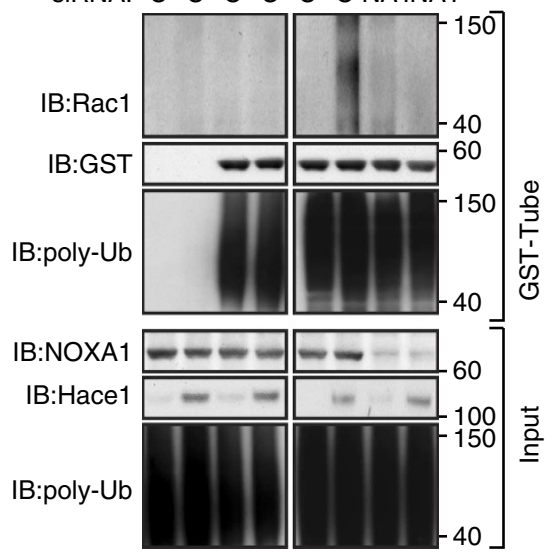

Figure 3 | Hace1 regulates the Nox1-containing NADPH oxidase. (a) MEFs derived from the Hace1 knockout $(-/-)$ mouse were transfected with HA-vector or HA-Hace1 in denoted combinations of GFP-vector, GFP-Rac1wt and GFP-Rac1-K147R for $48 \mathrm{~h}$ before incubated with the proteasome inhibitor MG132 for $2 \mathrm{~h}$, lysed in the presence of GST-TUBE2, subjected a GST pull-down and analyzed by immunoblotting for the indicated proteins. (b) Protein lysates from U2OS cells transfected with siRNA control (C), siRNA Rac1 (R), GFP-vector and GFP-Hace1 as indicated for $72 \mathrm{~h}$, were subjected to GFP:TRAP pull-downs of Hace1 interacting molecules and analyzed by immunoblotting for the indicated proteins. (c) U2OS cells transfected with HA-vector, HA-Hace1 in combinations with control (C) or NOXA1 (NA1) siRNAs for $72 \mathrm{~h}$, were incubated with $10 \mu \mathrm{M}$ MG132 and lysed with or without GST-TUBE2 as indicated, before being subjected to GST pull-downs and analyzed by immunoblotting for the indicated ubiquitylated proteins. (d) U2OS cells expressing empty vector (V), wild-type Hace1 (H), or C876S ligase-dead Hace1 (CS) were incubated with $10 \mu \mathrm{M}$ DHE for 30 min, analyzed for oxyethidium staining by a 595-nm enabled fluorescence microscope and quantified using ImageJ algorithm. (e) MEFs derived from wild-type (wt), Nox1-deficient (Nox1 - / - ) and Nox4-deficient (Nox4-/-) mice, were transfected with control ( $\mathrm{siC}$ ) or Hace1 (siH) siRNAs 72 hrs before being analyzed for ROS content as in c. For all panels when indicated, ${ }^{\star \star} P<0.01$ (Student's two-tailed $t$-test for equal variance); Error bars represent s.e.m. of at least three independent experiments. 
As GTP-loaded Racl binds NOXA1 at the complex to activate Nox1-containing NADPH oxidase ${ }^{30}$, we tested whether Hace1 also physically associates with NOXA1. Using a green fluorescent protein (GFP)-TRAP pull-down assay, NOXA1 and Rac1 were readily coimmunoprecipitated with GFP-Hace1. Knockdown of Racl using siRNAs dramatically inhibited the interaction between NOXA1 and Hace1 (Fig. 3b). This indicates that Hacel binds and targets Racl once the latter has been recruited to the complex. To validate this we analyzed whether NOXA1 is required for Rac1 ubiquitylation by Hace1. Indeed, siRNA-mediated NOXA1 knockdown completely blocked Hacel ubiquitylation of Rac1 (Fig. 3c), indicating that Hacel preferentially targets NOXA1associated Racl. Accordingly, the ability of Hacel to control ROS levels depend on its ligase activity, as expression of a ligase-dead C876S (CS) Hace1 mutant failed to reduce ROS (Fig. 3d). To obtain further genetic support for our model, we utilized MEFs derived from mice with targeted deletions of Nox1 $\left(\right.$ Nox $\left.1^{-/-}\right)$or Nox4 $\left(\mathrm{Nox}^{-/-}\right)$, as Nox1-containing NADPH oxidase complexes are Rac1-dependent while Nox4-containing complexes are Racl-independent ${ }^{32}$. While Hacel knockdown failed to increase ROS in Nox $1^{-/}$MEFs, it markedly did so in $w t$ and Nox4 $4^{-/-}$MEFs (Fig. 3e). These findings demonstrate that direct Hace1 targeting of NOXA1-bound Rac1 controls basal ROS levels generated by Racl-dependent NADPH oxidase complexes.

Hacel deficiency leads to NADPH oxidase-dependent DNA damage. We next wished to determine the physiological consequences of NADPH oxidase regulation by Hace1. As Hace $1^{-/-}$ mice are prone to tumorigenesis after IR or urethane treatment ${ }^{6}$, both of which induce DNA damage through ROS generation, we postulated that chronic elevated NADPH oxidase-mediated ROS might lead to increased DNA damage in Hace1-deficient cells. ROS-induced DNA damage response depends on ataxiatelangiectasia mutated (ATM) and the tumor suppressor p53, which are both phosphorylated in their active forms after DNA damage ${ }^{33,34}$. Exposure of Hace $1^{-/}$MEFs to low concentrations of exogenous $\mathrm{H}_{2} \mathrm{O}_{2}$ to simulate DNA damage markedly increased ATM and p53 Ser-15 phosphorylation compared with Hace ${ }^{+/+}$ cells, each of which was reduced by transient re-expression of $w t$ Hace1 (Fig. 4a; see GFP-Hace1 lanes). Low-dose IR also led to markedly higher phosphorylation of histone H2AX on serine 139 $(\gamma \mathrm{H} 2 \mathrm{AX})$, another well-established readout of DNA damage ${ }^{35}$, in Hace $1^{-/-}$versus Hace $1^{+/+}$MEFs (Fig. 4b,c,d), which again was reversed by transient wt Hacel re-expression in the former (Fig. 4d). The difference in $\gamma \mathrm{H} 2 \mathrm{AX}$ levels between Hace ${ }^{-/-}$ versus $\mathrm{Hace}^{+/+}$MEFs was already observed after $5 \mathrm{~min}$ of recovery from low-dose IR (Supplementary Fig. S3g), suggesting that this response is due to acute ROS generated by $\mathrm{IR}^{36}$. However, we cannot rule out whether DNA damage induced ROS amplification through other mechanisms, such as by direct H2AX activation of Nox $1^{37}$ may amplify the system. Similarly, in U2OS cells, exogenous $\mathrm{H}_{2} \mathrm{O}_{2}$-mediated ATM and $\gamma \mathrm{H} 2 \mathrm{AX}$ phosphorylation was blocked by transient Hacel overexpression (Fig. 4e), which reduces ROS in those cells (Fig. 2f). We then tested whether increased susceptibility to ROS-induced DNA damage in Hace1-deficient cells is dependent on Nox1-containing NADPH oxidase. Indeed, in Hace $1^{-/-}$MEFs, ML171 blocked $\mathrm{H}_{2} \mathrm{O}_{2}$-induced ATM phosphorylation (Fig. 4f) and Nox1 knockdown blocked exogenous $\mathrm{H}_{2} \mathrm{O}_{2}$-induced p53 Ser-15 phosphorylation (Fig. 4g). Moreover, while Hacel knockdown sensitized wt MEFs to $\mathrm{H}_{2} \mathrm{O}_{2}$-induced DNA damage, this was almost completely blocked in Nox1 MEFs exposed to $\mathrm{H}_{2} \mathrm{O}_{2}$ (Fig. 4h). Therefore, augmented DNA damage responses secondary to Hacel deficiency require
Racl-dependent NADPH oxidase activity. Finally, the human breast carcinoma cell line HCC1395, which lacks Hace1 expression due to a homozygous deletion of HACE1, had dramatically elevated $\gamma \mathrm{H} 2 \mathrm{AX}$ phosphorylation levels compared with HEK293 cells, even under ambient conditions (Fig. 4i). Finally, hace1 morpholino zebrafish showed a striking increase in $\gamma \mathrm{H} 2 \mathrm{AX}$ phosphorylation under basal conditions compared with $w t$ fish (Fig. 4j). These data provide compelling evidence that Hace1-deficient cells are hypersensitive to ROS-induced DNA damage.

Hacel controls cyclin D1 via regulation of ROS production. We previously showed that Hacel reduces in vitro and in vivo cell cycle progression of diverse Hace1-deficient tumor cell lines by repressing cyclin D1 protein expression, although Hace1 does not directly target cyclin D1 for degradation ${ }^{6}$. We therefore wondered if Hacel might also control cyclin D1 expression through its effects on Racl-dependent NADPH oxidases, particularly as induction of CCND1 (cyclin D1) messenger RNA (mRNA) expression has been linked to NADPH oxidase activity ${ }^{38}$. We first investigated Hacel regulation of ROS after mitogenic stimulation. Serum-starved Hace ${ }^{-1}$ MEFs displayed increased ROS compared with Hace ${ }^{+/+}$MEFs, and Hace ${ }^{-/-}$cells maintained elevated ROS accumulation in a time-dependent manner after serum re-stimulation (Supplementary Fig. S4a). This suggests that Hace ${ }^{-1-}$ controls ROS generation even under mitogenic stimulation and that reduced ROS in Hacel proficient cells is not simply a direct effect of decreased cell proliferation. Indeed, cyclin D1 levels were dramatically increased in Hace $1^{-/-}$compared with Hace $1^{+/+}$MEFs and this was inhibited by ML171 or APO (Fig. 5a). Moreover, ectopic Hace1 expression reduced cyclin D1 levels in $\mathrm{Hace}^{-/}$MEFs, which was further reduced by ML171 (Fig. 5b). Similarly, both Hace1 overexpression (Fig. 5c) and Racl siRNA knockdown (Fig. 5d) strongly reduced cyclin D1 expression in U2OS cells. Overexpression of the Racl-K147R mutant in these cells upregulated cyclin D1, which was reversed by ML171 (Fig. 5e). We then knocked down Hacel in $w t$, Nox1 $1^{-/}$and Nox4 $4^{-/-}$ MEFs; this increased cyclin D1 levels in $w t$ and Nox4 ${ }^{-/-}$MEFs, but failed to do so in Nox $1^{-/-}$MEFs (Fig. 5f). Therefore, Hace1deficient cells require Racl-dependent NADPH oxidase activity to induce cyclin D1 expression. We next analyzed ROS, Rac1 and cyclin D1 levels in a panel of Hace1-deficient human epithelial cancer cell lines (Fig. 5g). All cell lines displayed varying but significant increases in ROS, and this correlated strongly with elevated Rac1 and cyclin D1 levels compared with HEK293 cells (which express high Hacel levels ${ }^{6}$ ). Of note, Hacel mRNA and protein levels both increase in U2OS cells after acute ROS challenge with $\mathrm{H}_{2} \mathrm{O}_{2}$, and this correlates with increased binding and ubiquitylation of Racl by Hacel (Supplementary Fig. S4b-e). Hacel appears to bind only a small fraction of the total Racl pool, estimated to be $<10 \%$ (Supplementary Fig. S4f). This suggests that Hace1 expression and protein activity can be amplified by a ROS-dependent activation step to mediate its effect on Racl at membrane-associated NADPH oxidase complexes, and therefore on cyclin D1 expression.

Lastly, to test whether Hacel can block cell cycle progression in primary human cells through ROS regulation, freshly isolated human vein endothelial cells (HUVECs) were transfected with control or Hacel siRNAs and analyzed for ROS content. Hace1 knockdown markedly increased ROS levels in HUVECs as measured by DCFDA staining (Fig. 5h). Moreover, while the CNF1 Escherichia coli toxin, which activates Rac1 in HUVECs 9 , could induce cyclin D1 expression in these cells, cyclin D1 was constitutively induced in Hacel knockdown HUVECs even in the 
a

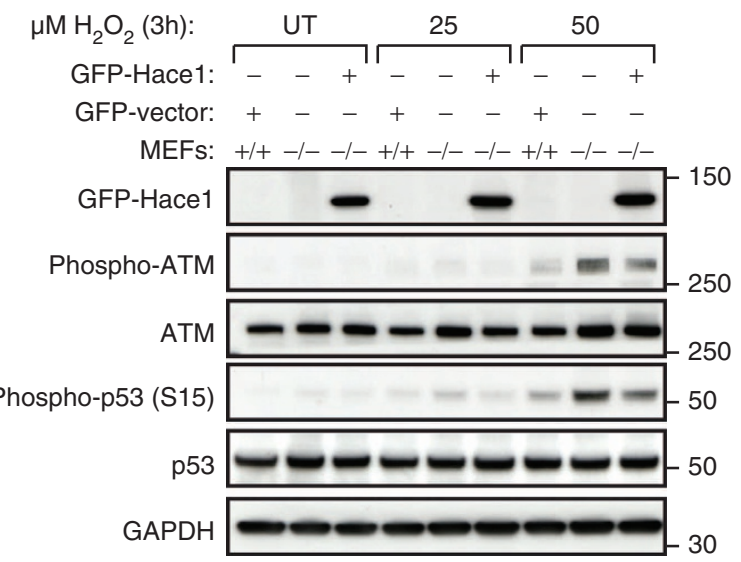

b

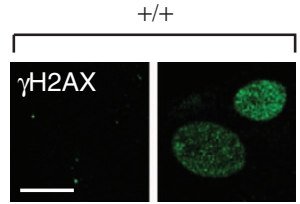

IR

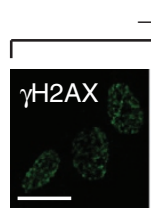

UT

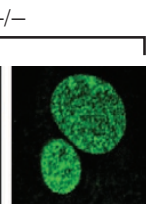

IR

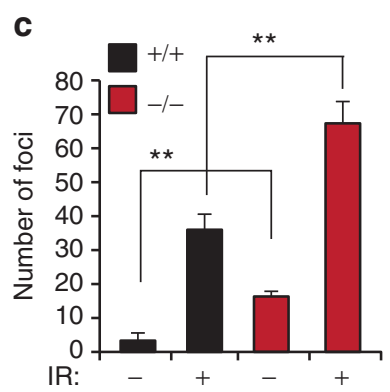

d

IR, 30 min: - $-\quad+\quad+\quad+$

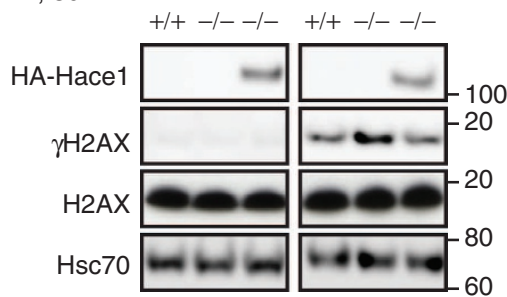

g

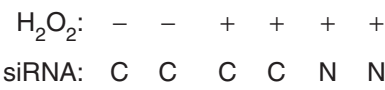

MEFs: $+/+-/-$ +/+ - /- +/+ - /-

Phospho-ATM

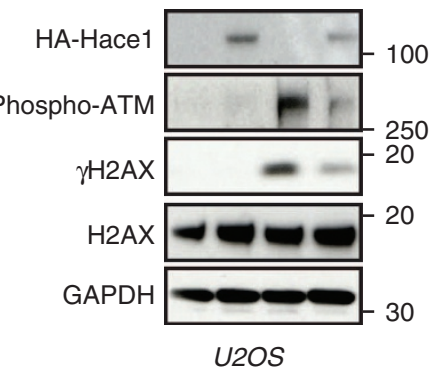

h
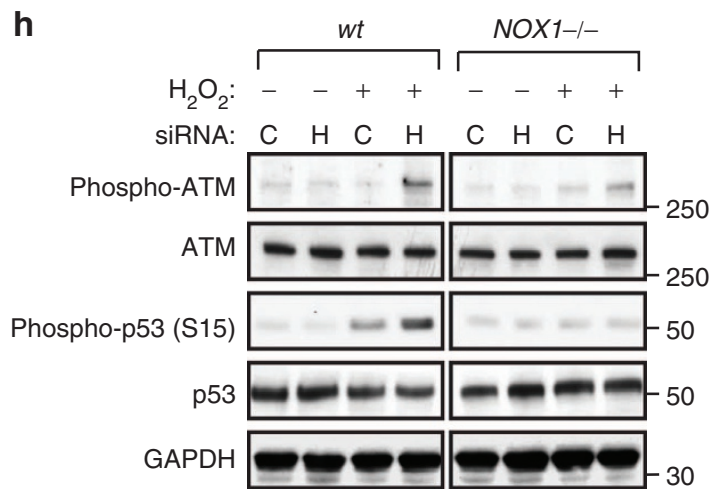

i

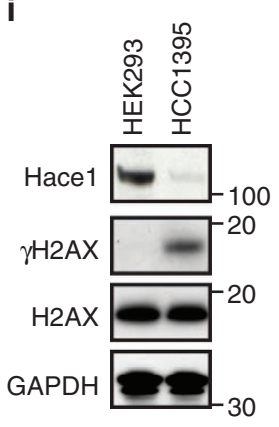

j
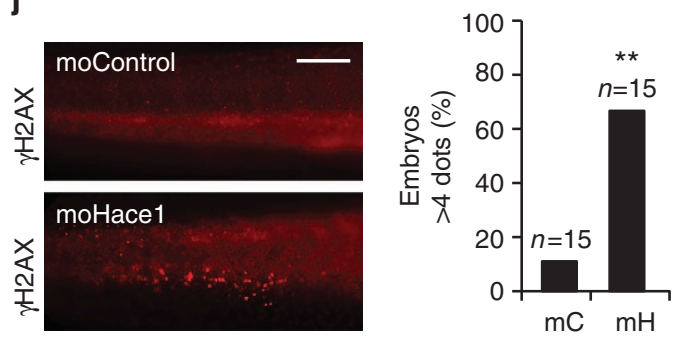

Figure 4 | Loss of Hace1 leads to Nox1-dependent activation of the DNA damage response. (a) MEFs derived from wild-type $(+/+)$ or Hace1 knockout $(-/-)$ mice were transfected with GFP-vector or GFP-Hace1 $48 \mathrm{~h}$ before being untreated (UT) or treated with indicated concentrations of $\mathrm{H}_{2} \mathrm{O}_{2}$ for $3 \mathrm{~h}$ and analyzed by immunoblotting for the indicated proteins. (b) MEFs derived as in a were subjected to 6 Gy IR for $1 \mathrm{~h}$ as indicated and analyzed for phosphorylated histone $\mathrm{H} 2 \mathrm{AX}(\gamma \mathrm{H} 2 \mathrm{AX}$ ) by immunostaining. Scale bar, $5 \mu \mathrm{m}$. (c) Quantification of $\gamma \mathrm{H} 2 \mathrm{AX}$ foci formation in high-power images obtained as in $\mathbf{b}$. (d) Lysates obtained from MEFs treated as in $\mathbf{b}$ were analyzed for expression of the indicated proteins by immunoblotting. (e) U2OS osteosarcoma cells transfected with HA-vector or HA-Hace1 for $48 \mathrm{~h}$ were left untreated or treated with $400 \mu \mathrm{M} \mathrm{H}_{2} \mathrm{O}_{2}$ for $3 \mathrm{~h}$ before being analyzed by immunoblotting for the indicated proteins. (f) MEFs derived as in a were treated with $10 \mu \mathrm{M} \mathrm{ML171}$ or $50 \mu \mathrm{M} \mathrm{H} \mathrm{H}_{2}$ as indicated and analyzed by immunoblotting for the indicated proteins. (g) MEFs derived as in a were transfected with control (C) or Nox1 (N) siRNAs, and treated with $50 \mu \mathrm{M} \mathrm{H}_{2} \mathrm{O}_{2}$ as indicated for $3 \mathrm{~h}$ and analyzed by immunoblotting for the indicated proteins. (h) MEFs derived from wild-type (wt) or Nox1 knockout (Nox $1^{-/-}$) mice were transfected with control (C) or Hace1 (H) siRNAs, treated with $50 \mu \mathrm{M} \mathrm{H}_{2} \mathrm{O}_{2}$ as indicated for $3 \mathrm{~h}$ and analyzed by immunoblotting for the indicated proteins. (i) $\mathrm{HEK} 293$ human embryonic kidney cells and HCC1395 breast cancer cells deficient for Hace1 expression were analyzed for phosphorylated histone H2AX ( $\gamma \mathrm{H} 2 \mathrm{AX}$ ) by immunoblotting. (j) Representative images (left) of $48 \mathrm{~h}$ post fertilization (hpf) casper zebrafish control $(\mathrm{mC})$ and hace $1(\mathrm{mH})$ morphants stained for phosphorylated histone $\mathrm{H} 2 \mathrm{AX}(\gamma \mathrm{H} 2 \mathrm{AX})$. The column graph (right) displays \% embryos $(n=15)$ with more than four $\gamma \mathrm{H} 2 \mathrm{AX}$ dots per tail. Scale bar, $0.25 \mathrm{~mm}$. For all panels when indicated, ${ }^{\star \star} P<0.01$ (Student's two-tailed $t$-test for equal variance); Error bars represent s.e.m. of at least three independent experiments.

absence of CNF1 (Fig. 5i). Importantly, Hacel knockdown correlated with increased $S$ and G2M fractions and cell proliferation of primary HUVECs (Fig. 5j), and co-knockdown of Nox1 decreased the fraction of cells in S-phase to control levels (Fig. 5k). Furthermore, the chemical Rac1 inhibitor EHT1864 efficiently reduced ROS in HUVECs with Hacel 
a

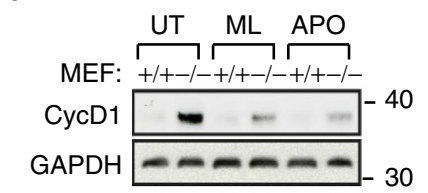

b

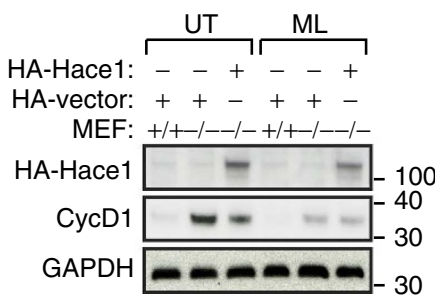

C

HA-Hace1: - + HA-vector: + -

HA-Hace $1 \square$

Cyclin D1 -30

GAPDH

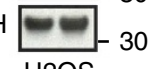

d

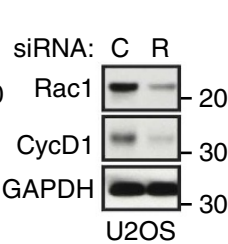

e

ML171: - - +

GFP-K147R: -++

GFP-vector: + - +

f
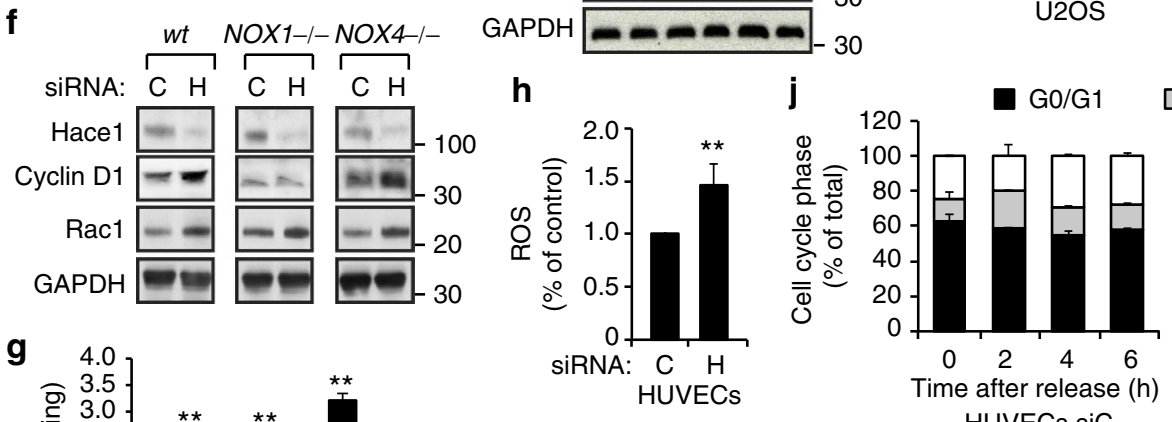

HUVECs siC

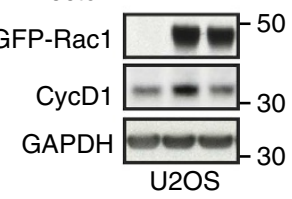

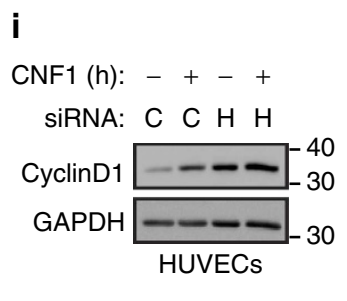

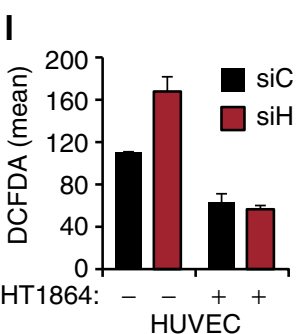

$\mathbf{n}_{\text {Serum: }-+\ldots+\ldots++}$

EHT1864: - + + + - + + siRNA: $\mathrm{C} \mathrm{C} \mathrm{C} \mathrm{C} \mathrm{H} \mathrm{H} \mathrm{H} \mathrm{H}$

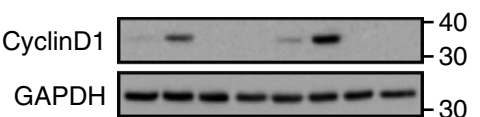

HUVEC

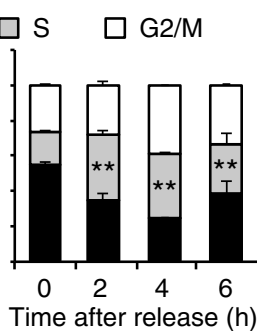

HUVECS SiH

m

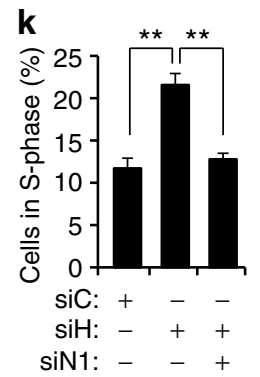

GAPDH

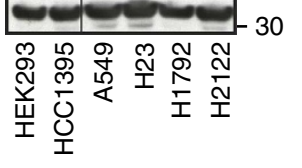

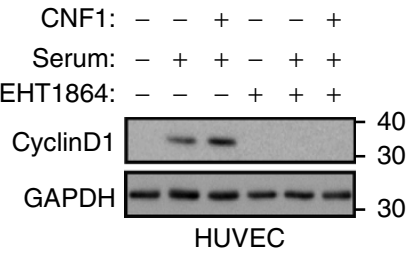

Figure 5 | Hace1 controls cyclin D1 expression by regulating of Nox1-containing NADPH oxidase. (a) Protein lysates from Hace1 wild-type ( $+/+$ ) or knockout $(-/-)$ MEFs were left untreated or treated overnight with ML171 (ML) or APO, were analyzed by immunoblotting as indicated. (b) MEFs as in a were transfected with Ha-vector or HA-Hace1, left untreated (UT) or treated overnight with ML171 (ML), and analyzed as in a. (c) U2OS cells transfected with HA-vector or HA-Hace1 were analyzed as in a. (d) U2OS cells transfected with control (C) or Rac1 (R) siRNAs were analyzed as in a. (e) U2OS cells transfected with GFP-vector or GFP-Rac1-K147R were left untreated or treated with ML171, and analyzed as in a. (f) Wild-type (WT), NOX4 knockout (NOX4-/-) or NOX1 knockout (NOX1-/-) MEFs were transfected with control (siC) or Hace1 (siH) siRNAs and analyzed as in a. (g) Indicated cell lines were analyzed for ROS by DHE staining and by immunoblotting of indicated proteins. (h) HUVEC cells were transfected with siRNA control (C) or Hace1 (H) and analyzed for ROS content by DCFDA assay. (i) HUVEC cells transfected as in $\mathbf{h}$ were analyzed for the indicated proteins by immunoblotting after stimulation with vehicle or CNF1 $\left(10^{-9} \mathrm{M}\right)$. (j) HUVEC cells transfected as in $\mathbf{h}$ were analyzed for cell cycle distribution (G1, S, G2/M phase) after release from a thymidine cell cycle block at the indicated time points. (k) HUVEC cells transfected with siRNA control (C), Hace1 $(\mathrm{H})$ or Nox1 (N1) as in $\mathbf{h}$ were analyzed for cells in S-phase after release from a thymidine cell cycle block. (I) HUVEC cells transfected with siRNA control ( $\mathrm{siC}$ ) or Hace1 ( $\mathrm{siH}$ ) were stimulated as in $\mathbf{i}$, treated with EHT1864 as indicated and analyzed for ROS content as in $\mathbf{h}$. (m) Lysates from HUVEC cells synchronized in GO by serum deprivation and stimulated with serum for $15 \mathrm{~h}$ with or without EHT1864 and stimulation with vehicle or CNF1 were analyzed as in a. (n) Lysates from control (C) or HACE1 (H) knocked down HUVEC cells treated as in $\mathbf{m}$ were analyzed as in a. For all panels when indicated, ${ }^{\star \star} P<0.01$ (Student's two-tailed $t$-test); Error bars: s.e.m. of at least three independent experiments.

knockdown (Fig. 5l) and abolished cyclin D1 induction by CNF1 or serum (Fig. 5m). Finally, EHT1864 blocked serum-stimulated cyclin D1 induction in HUVECs with Hacel knockdown (Fig. 5n). Collectively, these data suggest that Hacel controls cyclin D1 expression and cell cycle progression through its ability to block activity of Rac1-dependent NADPH oxidase complexes.

Increased ROS in Hace1-deficient murine and human tumors. To demonstrate the in vivo significance of the above findings, we examined tissues from Hace $1^{-/-}$mice, which develop diverse spontaneous late-onset tumors across all three germ layers ${ }^{6}$. Liver tumors from these mice showed strikingly higher ROS levels than matching normal liver tissue from the same mice, or from Hace $^{+9+}$ littermates (Fig. 6a). Hace1 loss strongly correlated with elevated Rac1, cyclin D1 and phosphorylated Ser-15-p53 levels in tumor lysates (Fig. 6b). This was also observed in Hace 1 - / - mouse colorectal carcinoma as compared to normal tissue (Fig. 6c). This indicates that not only do normal tissues of Hace1-/ - mice have higher ROS levels compared with wt mice (Fig. 1e), but there is further ROS accumulation in the tumors, possibly due to clonal selection during transformation. 
a

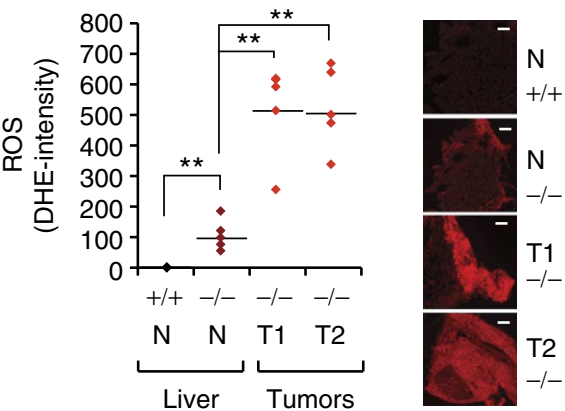

b

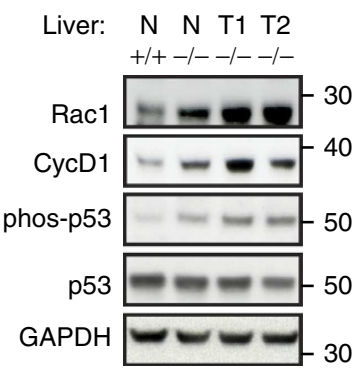

C

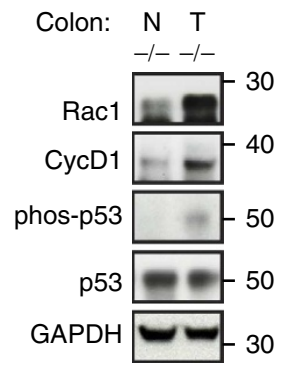

d

Patient 1

Patient 2

Patient 3
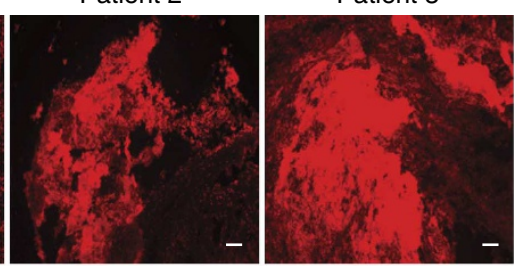

Patient 4

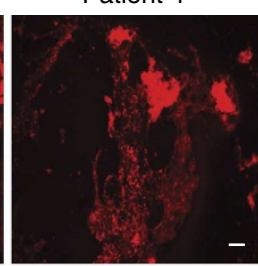

Patient 5
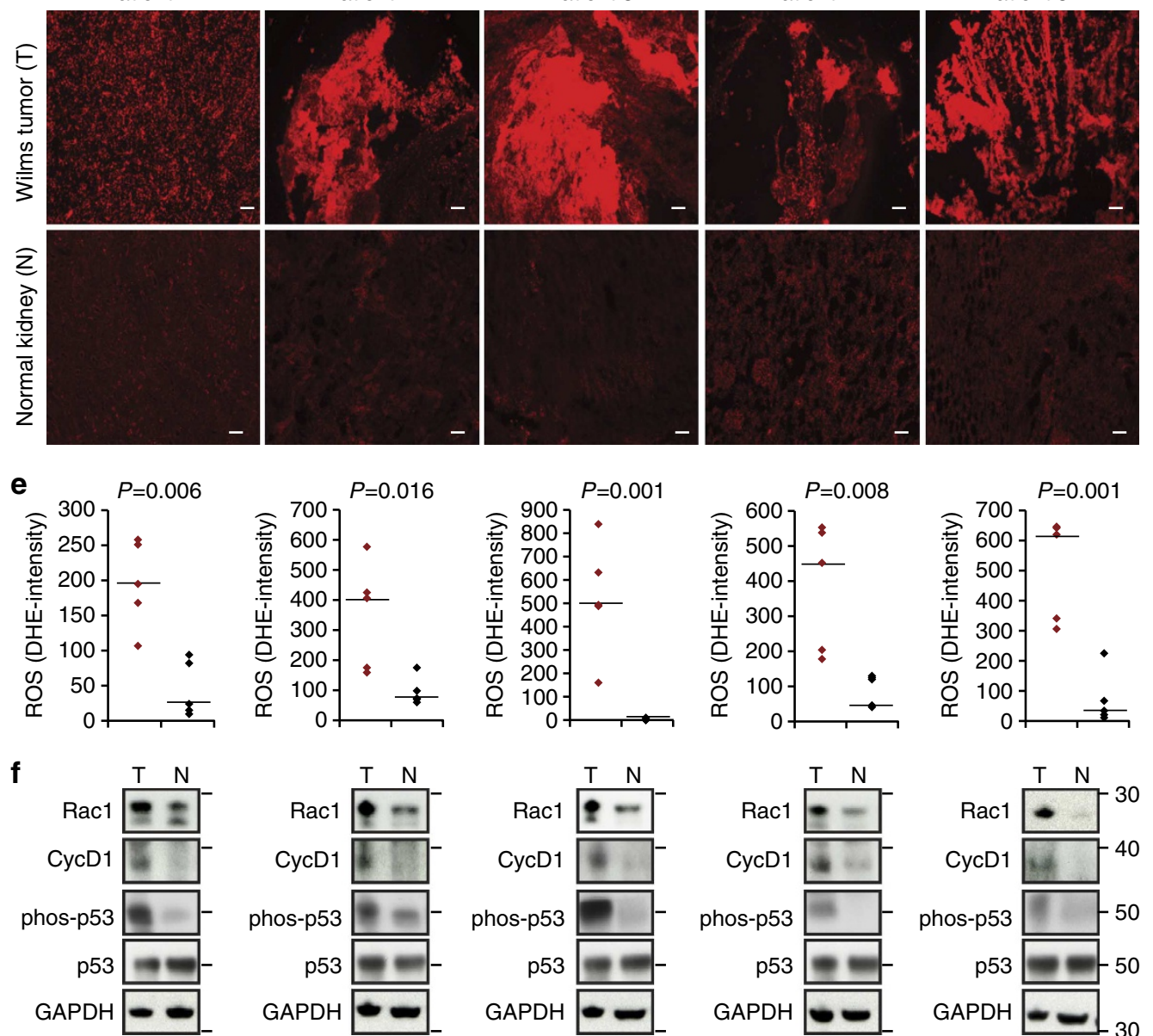

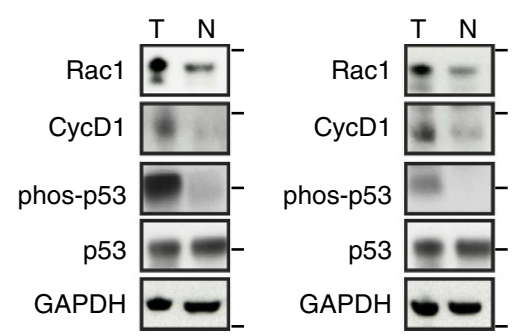

Figure 6 | Characterization of murine and human Hace1-deficient tumors. (a) Snap-frozen samples of normal liver $(N)$ from wild-type $(+/+)$ and Hace $1-/-$ mice $(-/-)$ and matching spontaneous liver tumor tissues (T1 and T2) from the same Hace $-/-$ mice were cut in $20 \mu \mathrm{m}$ sections using a cryostat at $-20^{\circ} \mathrm{C}$, incubated with $10 \mu \mathrm{M} \mathrm{DHE}$ for $30 \mathrm{~min}$ and analyzed for oxyethidium staining by a 595-nm enabled fluorescence microscope (right) and quantified using ImageJ algorithm (left). Scale bars, $20 \mu \mathrm{m}$. Horizontal lines in the graphs represent median values of quantification by ImageJ algorithm. ${ }^{\star \star} P>0.01$ (Student's two-tailed $t$-test for equal variance). (b) Protein lysates isolated from tissues shown in a were analyzed for expression levels of the indicated proteins by immunoblotting. (c) Protein lysates from normal colon tissue $(N)$ and colonic tumor tissue (T) dissected from Hace1 knockout $(-/-)$ mice were analyzed for the expression of the indicated proteins by immunoblotting. (d) Snap-frozen tissue of matched normal kidney

$(\mathrm{N})$ and Wilms' tumors (T) from the same patients were analyzed for ROS as in a. Scale bars, $20 \mu \mathrm{m}$. (e) High power field images obtained as in $\mathbf{d}$ were quantified for DHE signal intensity (ROS content) by ImageJ software. Lines represent median values. (f) Lysates from tissue as in $\mathbf{d}$ were analyzed for expression of the indicated proteins (lower panels) by immunoblotting.

As Hace1 was originally discovered as a tumor suppressor in human Wilms' tumor ${ }^{1,6}$, we next investigated a panel of patientmatched Wilms' tumor and normal kidney specimens, each previously documented to have lost Hacel protein expression ${ }^{1}$. All tumors analyzed displayed markedly increased ROS (Fig. 6d,e), which correlated with elevated Rac1 and cyclin D1 expression, as well as phosphorylated Ser-15-p53 levels (Fig. 6f). This clearly demonstrates that Hacel-deficient mouse and human tumors have chronic high ROS, elevated Racl and cyclin D1 expression, which is coupled with increased DNA damage response signaling, confirming the in vivo significance of our findings. 


\section{Discussion}

ROS are essential for cell homeostasis and has diverse roles in health and disease. Along with the mitochondria, which continuously generate ROS as byproducts of oxidative phosphorylation $^{39}$, a major source of cellular ROS are the NADPH oxidases $^{21}$. These enzymes utilize molecular oxygen and NADPH to produce superoxide, which is then rapidly converted to cellular $\mathrm{H}_{2} \mathrm{O}_{2}$ by superoxide dismutase enzymes ${ }^{21}$. NADPH oxidase complexes are found in all multicellular organisms where they are solely dedicated to the generation of ROS. In non-pathological settings, ROS functions as a second messenger for processes such as differentiation, proliferation and cell motility ${ }^{40}$. In pathologic conditions, however, ROS can oxidize biomolecules such as DNA, causing DNA damage and is likely the greatest intrinsic threat to genome integrity. Accordingly, organisms have evolved a tightly regulated cellular response to acute oxidative stress to detoxify ROS and repair damaged $\mathrm{DNA}^{33}$. While cellular detoxification systems are well-characterized ${ }^{41}$, little is known about the regulation of ROS generation by NADPH oxidases. Here we identify the tumor suppressor Hacel as a highly conserved direct negative regulator of Racl-dependent NADPH oxidases through degradation of complex-bound Rac1. Hacel deficiency leads to a deregulated and hyperactive ROS-generating holoenzyme that maintains a state of chronic high ROS in vitro and in vivo. This is associated with ROS-induced DNA damage and cyclin D1 expression, both of which are blocked by pharmacologic or genetic inactivation of Nox1-containing NADPH oxidases. Based on these observations, we propose a model whereby Hace1 recruitment to Rac1-containing NADPH oxidases leads to Rac1 degradation and inactivation of the complex (Fig. 7). Together with Rac1 guanine nucleotide exchange-factors ${ }^{42}$, Hace1mediated ubiquitylation of Racl therefore adds another layer of control to the regulation of the NADPH oxidase system. These results highlight a novel regulatory pathway that protects cells from ROS-induced DNA damage and cyclin D1-mediated hyperproliferation. This is the first known example, to our knowledge, of an E3 ligase that directly regulates NADPH oxidase activity and of a tumor suppressor that directly regulates ROS generation in vertebrate cells.

Activation of Rac1-dependent NADPH oxidase complexes requires the binding of GTP-loaded Racl to the NADPH oxidase
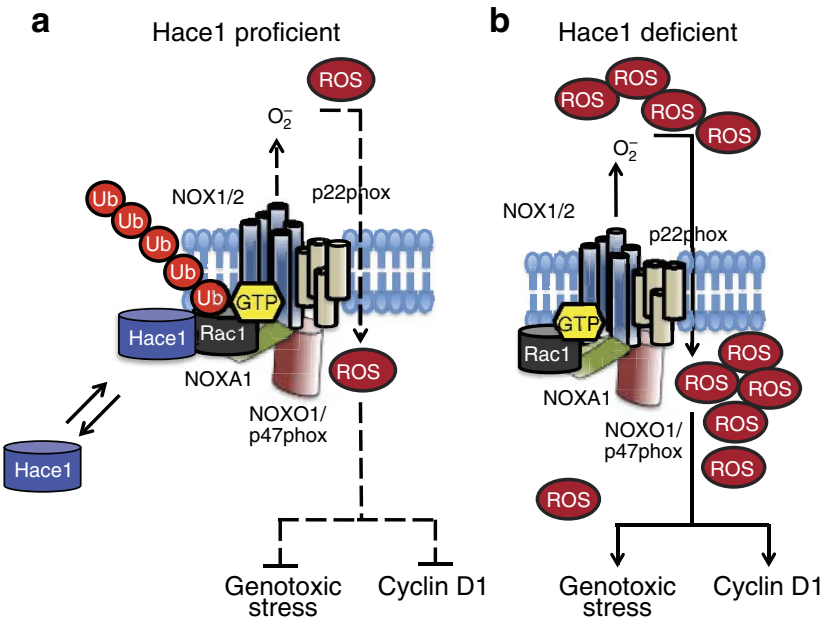

Figure 7 | Hace1 controls NADPH oxidase. (a) Hace1 targets complexbound Rac1 to regulate NADPH oxidase, ROS production, cyclin D1 expression and DNA damage susceptibility. (b) Loss of Hace1 leads to hyperactive NADPH oxidase activity, increased ROS generation, high cyclin D1 expression and ROS-induced DNA damage. subunit, Noxa1. Mechanistically, we show that Hacel binds and ubiquitinates Rac1 when the latter associates with the complex through Noxa1. This then targets complex-bound Rac1 for proteasomal degradation and inhibits NADPH oxidase activity. Whether Hacel requires an activation step before it can bind Racl at the complex is currently unknown. HACE1 has recently been implicated as a target gene of the Nrf 2 transcription factor ${ }^{43}$. Nrf2 regulates transcription of a wide spectrum of genes involved in the oxidative stress response ${ }^{43}$. Indeed, Hacel mRNA and protein levels are increased in U2OS cells after acute ROS challenge with $\mathrm{H}_{2} \mathrm{O}_{2}$. It is therefore tempting to speculate that Hacel becomes activated by oxidative stress to subsequently shut down one of the main ROS-generating enzymes in cells as a part of the general oxidative stress response.

While somewhat contentious, the current view is that Nox1, Nox2 and Nox3 containing NADPH oxidase complexes are clearly Rac1-dependent for activation, while Nox4, Nox5 and DUOX containing complexes are Racl-independent ${ }^{21,44}$. Nox1 is thought to be the major Nox subunit of NADPH oxidases in epithelial and mesenchymal cells, while Nox2 expression is more restricted to hematopoietic cells ${ }^{21}$. However, Nox2 may have broader expression in epithelial and mesenchymal cells, while Nox3 is expressed only at low levels in these cell types (ref. 31). Our studies point to a general role for Hacel in regulating Rac1-containing NADPH oxidase complexes as it blocks ROS generated from both Nox1- and Nox2-containing complexes. As Hacel only binds a small fraction of the total Racl pool, estimated as $<10 \%$ and mainly associated with NADPH oxidase, it is unknown whether Hacel targets other subcellular pools of GTP-Rac1 under context-specific conditions.

Ros can be pro-oncogenic as a consequence of increased replication stress and incomplete repair of ROS-induced DNA damage. Racl and Noxl are required for cellular transformation by numerous oncogenes including activated K-Ras, $\mathrm{H}$-Ras, c-Met, c-Myc and c-Src, and oncogene transformation is consistently associated with elevated ROS levels ${ }^{45-49}$. While the role of ROS in transformation is incompletely understood, elevated ROS produced by oncogenic signaling may contribute to the accumulation of additional mutations, further supporting tumor progression. Disruption of the Hace1-Rac1-NADPH oxidase axis described here may therefore impact a potentially broad spectrum of human tumors.

As ROS scavenging by anti-oxidants inhibits the transformed phenotype $\mathrm{e}^{50}$, transformed cells may become addicted to chronic high ROS levels to maintain specific aspects of pro-oncogenic signaling. Interestingly, induction of CCND1 (cyclin D1) mRNA expression by the AP-1 transcription factor is known to require NADPH oxidase activity ${ }^{38}$. Indeed, we demonstrate that cyclin D1 induction in Hace1-deficient cells also depends on intact Rac1 and NADPH oxidase activity. D-type cyclins (D1 and D3 in particular) have recently been linked to the maintenance of tumor growth in both epithelial and hematopoietic malignancies ${ }^{51,52}$. Therefore, the NADPH oxidase and ROS-driven cyclin D1 expression observed in Hace1-deficient cells might contribute broadly to progression of spontaneous tumors. We found that the EHT1864 Rac1 inhibitor strongly blocks ROS-induced cyclin D1 expression, a finding that may have clinical implications. Also, as ROS has pathogenic roles in other human diseases such as cardiac hypertrophy and ischemia-reperfusion injury, immune system disorders, liver disease, diabetes, obesity, atherosclerosis, Alzheimer's disease, Parkinson's disease, Huntington disease, rheumatoid arthritis, aging and HIF1/ hypoxia-related diseases ${ }^{53-56}$, further studies of pathogenic Hacel deficiency may reveal additional disease links.

In summary, we find that Hacel deficiency leads to chronic elevated cellular ROS and ROS-induced DNA damage response 
signaling, which is blocked by pharmacologic or genetic inactivation of Rac1-containing NADPH oxidases. These results identify Hacel as a de facto negative regulator of NADPH oxidase activity in vertebrates and highlight a novel regulatory pathway that protects cells from ROS-induced DNA damage. It will be important to determine how this specific function of Hacel contributes to its tumor suppressor activity, and whether Hacel deficiency has roles in other pathologic conditions.

\section{Methods}

Cell culture and reagents. All cells were grown in DMEM (Dulbecco) supplemented with $10 \%$ fetal calf serum, non-essential amino acids and penicillinstreptomycin. Wild-type $(w t)$ and Hacel knockout MEFs were derived from BL6 WT and Hace1 - / - littermates and allowed to spontaneously immortalize. WT, Nox $1-/$ - and Nox4 - I - MEFs were a kind gift from Dr Denis Martinvalet, University of Geneva. HUVEC cells were derived as described in Torrino et al. ${ }^{9}$. DNA transfections were performed with FugeneHD for human cell lines and with electroporation using Neon Transfection System (Invitrogen) for mouse cell lines. MSCV-HA, MSCV-HA-Hacel and MSCV-HA-Hace1-C876S were cloned as described in Zhang et al. ${ }^{6}$. GFP-Hacel and GFP Hacel-C876S were generated by cloning Hacel and C876S into pEGFP-C1 (Clontech). GFP-Rac1wt, GFP-Rac-1v12 were generated as described in Castillo-Lluva et al. ${ }^{57}$ and GFP-Rac1-K147R was made by PCR-based nucleotide mutagenesis in GFP-Rac1. Transfections of siRNAs were performed with $25 \mathrm{nM}$ siRNA using RNAiMax (Invitrogen). The following siRNAs were used in the study: Control siRNA (C): $\left(5^{\prime}-3^{\prime}\right)$ AUAUCGGCUAG GUCUAACA; Hace1-1 (H1): Hs_Hacel_1 (FlexiTube, Qiagen); Hace1-2 (H2) Hs_Hacel_4 (FlexiTube, Qiagen); Human Racl (R): Hs_Rac1_6 (FlexiTube, Qiagen); Human Nox2 (N2): Hs_CYBB (FlexiTube, Qiagen); Murine Rac1-1 (R1): Mm_Rac1_1 (FlexiTube, Qiagen); Murine Rac1-2 (R2): Mm_Rac1_2 (FlexiTube, Qiagen); Murine Rac1-3 (R3): Mm_Rac1_3 (FlexiTube, Qiagen); Murine Rac1-4 (R4): Mm_Rac1_8 (FlexiTube, Qiagen). Human and mouse Nox1, human NoxA1, human and mouse NOXO1 and mouse p22phox siRNA ON-TARGETplus SMARTpools were obtained from Dharmacon. Camptothecin, hydrogen peroxide $\left(\mathrm{H}_{2} \mathrm{O}_{2}\right)$, DPI, 2-acetylphenothiazine (ML171), APO, DHE, EHT1864 and MG132 were purchased from Sigma. Piperlongumine (PL) was purchased from RD Chemicals. IR was performed in an X-ray generator (Pantak HF160, $150 \mathrm{kV}$, $15 \mathrm{~mA}$, dose rate 2.18 Gy per minute).

Reactive oxygen species measurements. Crude ROS levels were measured by CM-H2-DCFDA (Invitrogen) according to the manufacturer's guidelines. Primary cells (HUVECs) were incubated with $5 \mathrm{mM} \mathrm{2}, 7^{\prime}$-dichlorodihydrofluorescein diacetate (DCFDA, Santa-Cruz) for $20 \mathrm{~min}$ at $37^{\circ} \mathrm{C}$. Cells were washed twice in PBS, trypsinized and fluorescence was measured using flow cytometry. For each experiment, fluorescence was determined on triplicate samples. Statistical analysis of the results was performed with Prism 5.0b (GraphPad software) by one-way ANOVA with Bonferroni post hoc. Superoxide levels were measured using the DHE probe. In the presence of the superoxide anion $\mathrm{O}_{2}^{-}$, DHE is rapidly oxidized to oxyethidium, which binds DNA and emits light in the $570-580 \mathrm{~nm}$ ranges when excited at $488 \mathrm{~nm}$. For cell culture, after appropriate treatments, cells were washed in Hank's Balanced Salt Solution (HBSS), incubated for 30-60 min in HBSS containing $10 \mu \mathrm{M}$ DHE, washed in HBSS and directly analyzed for oxyethidium fluorescence with an epi-fluorescence HAL100 microscope (Zeiss). For tissue sections, snap-frozen tissue samples and tumors were cut in $20 \mu \mathrm{m}$ sections using a cryostat, washed and DHE-treated as described for cell lines, mounted on cover slides and analyzed as for cell lines. When indicated, oxyethidium emission was analyzed and quantified using ImageJ software. For all specimens, haematoxylin and eosin staining were performed side-by-side to verify tissue integrity and pathology, using standard methods. NTB was used to detect localized ROS in vitro. When NTB becomes oxidized it creates an insoluble blue formazan dye detectable using a high-resolution light microscope. Exponentially growing cells were incubated with a saturated NTB solution (in $\mathrm{H}_{2} \mathrm{O}$ ) for $30 \mathrm{~min}$ in $37^{\circ} \mathrm{C}$ before washed $2 \times$ in PBS, air-dried and analyzed in a $60 \times$ magnification light microscope.

Immunodetection. For immunoblotting, proteins separated by SDS-polyacrylamide gel electrophoresis (PAGE) and transferred to a nitrocellulose membrane were detected with indicated primary and appropriate secondary antibodies, enhanced chemiluminescence (ECL) western blotting reagents (Thermo Scientific), and film (Kodak). Primary antibodies used in the indicated dilutions were purchased from Sigma (Hacel (1:1,000), NOXA1 (1:500), Noxl (1:300), Roche (GFP (1:2,000)), Rockland (phospho-S1981-ATM (1:1,000)), Cell Signaling (ATM (1:1,000), p53 (1:1,000), phosphoS15-p53 (1:1,000), phosphoS139-H2AX (1:1,000), H2AX (1:1,000), GAPDH $(1: 2,000)$, cyclin D1 (1:1,000)), BD Biosciences (Rac1 (1:500)), Santa-Cruz (Rac1 (1:1,000), p22phox (1:250), p67phox (1:500), p47phox (1:500), GST (1:1,000), Nox2 (1:500)), ENZO (poly-ubiquitin (1:1,000)), Assay Designs (Hsc70 (1:1,000)) and Covance (HA $(1: 1,000))$. Secondary horseradish peroxidase-conjugated antibodies (1:10.000) used for immunoblotting were purchased from DAKO. Full-length scans of immunoblots with key data are shown in Supplementary Fig. S5.
GFP:TRAP and TUBE pull-down assays. For GFP:TRAP pull-downs, whole-cell lysates were dissolved in TRAP-buffer (10 mM Tris-HCL pH 7.5, 0.5\% NP-40, $150 \mathrm{mM} \mathrm{NaCl}, 0.5 \mathrm{mM}$ EDTA) supplemented with $1 \mathrm{nM}$ PMSF (Active Motif) protease and phosphatase inhibitors (Roche). Clarified extracts were incubated for $3 \mathrm{~h}$ in a rotator at $4^{\circ} \mathrm{C}$ with $30 \mu \mathrm{l}$ GFP-Trap_A beads (Chromotech). Precipitated immunocomplexes were washed $5 \times$ in TRAP-buffer without NP-40, boiled in SDS sample buffer containing $50 \mathrm{mM}$ dithiothreitol, and analyzed by immunoblotting.

TUBEs were used to purify endogenous ubiquitin conjugates from cell lysates according to the manufacturer's recommendations with minor modifications. Briefly, lysis buffer $\left(20 \mathrm{mM} \mathrm{Na}_{2} \mathrm{HPO}_{4}, 20 \mathrm{mM} \mathrm{NaH}_{2} \mathrm{PO}_{4}, 1 \% \mathrm{NP}-40,2 \mathrm{mM}\right.$ EDTA) was supplemented with $1 \mathrm{mM}$ dithiothreitol, $1 \times$ protease inhibitor mix (Sigma) and $50 \mu \mathrm{g} \mathrm{ml}^{-1}$ of GST-TUBE2 (Lifesensors, Malvern, PA). One $70-80 \%$ confluent $10 \mathrm{~cm}$ dish per condition was treated as indicated, after which cells were scraped off the dish in PBS and pelleted by centrifugation. Cells were lysed in $300 \mu \mathrm{L}$ lysis buffer and kept on ice for $20 \mathrm{~min}$ and lysates were cleared by centrifugation. Approximately $5 \%$ of the cleared lysates was used for input and the remaining lysate was added to $20 \mu \mathrm{l}$ of washed Glutathione Sepharose 4 Fast flow beads (GE Healthcare) for capture of GST-TUBE2 and bound material. Reactions were kept at $4{ }^{\circ} \mathrm{C}$ with rotation for $1-2 \mathrm{~h}$, followed by $4 \times$ washing in $500 \mu \mathrm{l}$ of icecold TBS containing $0.1 \%$ Tween 20 (TBS-T). Bound material was separated from beads by addition of $1 \times$ LSB and analyzed on SDS-PAGE for the indicated proteins.

PCR assay. PCR was used to detect the expression of Nox gene expression in SKOV3 cells. RNA was purified according to standard protocol (Qiagen) and reverse transcribed into complementary DNA (cDNA; Qiagen). PCR was performed in $50 \mu \mathrm{l}$ of $10 \mathrm{mmoll}^{-1}$ Tris- $\mathrm{HCl}\left(\mathrm{pH} \mathrm{8.3)}, 25 \mathrm{mmoll}^{-1} \mathrm{MgCl}_{2}\right.$, $10 \mathrm{mmoll}^{-1} \mathrm{dNTP}, 100 \mathrm{U}$ of Taq DNA polymerase, with $0.1 \mu \mathrm{moll}^{-1}$ each primer and was terminated by heating at $70^{\circ} \mathrm{C}$ for $15 \mathrm{~min}$. PCR products were resolved on a $1 \%$ agarose gel and visualized with ultraviolet light after ethidium bromide treatment. The following primers ware used $\left(5^{\prime}-3^{\prime}\right)$ : human Noxl sense, CTTCCTCACCGGATGGGACA; human Nox1 antisense, TGACAGCATTTGCG CAGGCT; human Nox2 sense, CCTGTACCTGGCTGTGACCCTGTT; human Nox2 antisense, ACCCCAATCCCTGCTCCCACTAA; human Nox3 sense, GAGTGGCACCCCTTCACCCT; human Nox3 antisense, CTAGAAGCTCTCCTT GTTGT; human Nox4 sense, AGTCAAACAGATGGGATA; human Nox4 antisense, TGTCCCATATGAGTTGTT; human Nox5 sense, AAGCATACTTG CCCCAGCTG; human Nox5 antisense, CAGGCCAATGGCCTTCATGT.

Microscopy. All microscopy was performed on a HAL100 Zeiss microscope Image acquisition and analysis was carried out with LSM-ZEN software and ImageJ.

Zebrafish Hace1 model. Zebrafish were maintained, bred and developmentally staged as described ${ }^{58}$. The Dalhousie University Animal Care Committee approved use of zebrafish in this study. The hace1-HECT morpholino (5'-CCCTCGAAC TGTTAGACAGAATAAA)- $3^{\prime}$ and standard control morpholino (5'-CCTCTTA CCTCAGTTACAATTTATA) $-3^{\prime}$ were purchased from Genetools LLC (Philomath, OR). The hace1-HECT morpholino targets a splice site which resides within the catalytically active HECT domain, and is positioned upstream of the critical cysteine residue (C876S) required for hacel ubiquitin ligase function. Morpholinos were diluted to a working concentration of $1.6 \mathrm{mM}$ with $1 \%$ phenol red, and were injected into live zebrafish embryos at the 1-4 cell stage. Digoxigenin- and fluorescein isothiocyanate (FITC)-labeled antisense RNA probes were transcribed from linearized cDNA constructs according to the manufacturer's protocols (Roche Molecular Biochemicals, Indianapolis, IN). Whole-mount in situ hybridization assays for zebrafish embryos were conducted as described ${ }^{59}$. Staining was performed using BCIP/NBT (Vector laboratories, Burlington, ON, Canada). The Hacel morpholino was verified by reverse transcription-PCR using the following primers $\left(5^{\prime}-3^{\prime}\right)$ : Primer A: forward $5^{\prime}$-TTGCTGGTCAAATCCTGGGTCTGG-3'; Reverse-5'-AATGCAGTGCGACAAGCAAGCG-3'; Primer B: Forward-5'-AGCC AGGAGGAACTACCATTCAGG-3'; Reverse-5'-ATAACTCCCACAATGCAG TGCGAC- $3^{\prime}$. Single Whole-mount in situ hybridization images were taken on a Leica MZ16F with a DFC 490 camera. Double fluorescence in situ experiments were performed with Fast Red (Vector laboratories) staining, and then imaged with a Zeiss Observer Z.1 microscope with a Colibri fluorescence source. $\mathrm{H}_{2} \mathrm{O}_{2}$ imaging using a live cell fluorescein dye was adapted from a previously described assay ${ }^{60}$. In brief, $48 \mathrm{~h}$ post fertilization (hpf) casper embryos were loaded for $30 \mathrm{~min}$ with $50 \mu \mathrm{M}$ pentafluorobenzenesulphonyl fluorescein (Cayman Chemical) in 1\% dimethylsulphoxide in egg water, and imaged using a 550-nm bandpass filter. Where indicated, the morpholinos were incubated for $1 \mathrm{~h}$ with $100 \mu \mathrm{M}$ DPI or $24 \mathrm{~h}$ with $200 \mu \mathrm{M}$ APO. Zebrafish embryos were dissociated to a single-cell suspension, and sorted on a FACSAria I (BD Biosciences, San Jose, CA, USA). Cells were thresholded by forward scatter (FSC) and side scatter (SSC) and interrogated by Coherent Sapphire solid state $488 \mathrm{~nm}$ laser with dual bandpass filter including FITC $(515-545 \mathrm{~nm})$ emission. ROS $^{+}$cells were gated by the following parameters: $\mathrm{FSC}^{\mathrm{LO}}$ and $\mathrm{SSC}^{\mathrm{LO}}$, and FITC ${ }^{\mathrm{HI}}$ fluorescence. 
Statistical tests. When indicated, data were subjected a two-tailed Student's $t$-test to resolve statistical significance. Data compiled from several experiments are presented with a \pm s.e.m.

Human and murine tissue material. Snap-frozen matched tissue specimens of human normal kidney and Wilms' tumor from the same patients were obtained from the Children's Oncology Group Wilms' tumor tissue bank, kindly contributed by Dr Paul Grundy, Cross Cancer Centre, Edmonton, Alberta. Snap-frozen murine tumors were obtained from wild-type and Hacel knockout mice at the BC Cancer Research Centre. The British Columbia Cancer Agency and UBC ethical committees have approved the use of human tissue specimens and mouse models in this study. All specimens were cut in $20 \mu \mathrm{m}$ sections at $-20^{\circ} \mathrm{C}$ using a cryostat, mounted on cover slides on dry ice, washed in ice-cold HBSS, and immediately DHE-treated as described for cell lines and analyzed for oxyethidium light emission by microscopy. In parallel, $5 \mu \mathrm{m}$ sections were cut and haematoxylin and eosin stained for verification of tissue integrity and pathology. All tumor sections con tained at least $80 \%$ viable tumor tissue. In cases with sufficient tissue available, additional sections were cut for preparing protein lysates to be analyzed by SDSPAGE for the indicated proteins. Wilms' tumors used in this study were chosen on the basis of having been previously shown to have low or undetectable Hacel mRNA and protein expression as measured by reverse transcription-PCR or western blotting, respectively ${ }^{6}$.

\section{References}

1. Anglesio, M. S. et al. Differential expression of a novel ankyrin containing E3 ubiquitin-protein ligase, Hace1, in sporadic Wilms' tumor versus normal kidney. Hum. Mol. Genet. 13, 2061-2074 (2004).

2. Diskin, S. J. et al. Common variation at $6 \mathrm{q} 16$ within HACE1 and LIN28B influences susceptibility to neuroblastoma. Nat. Genet. 44, 1126-1130 (2012).

3. Hibi, K. et al. Aberrant methylation of the HACE1 gene is frequently detected in advanced colorectal cancer. Anticancer Res. 28, 1581-1584 (2008)

4. Sakata, M. et al. Methylation of HACE1 in gastric carcinoma. Anticancer Res. 29, 2231-2233 (2009)

5. Slade, I. et al. Constitutional translocation breakpoint mapping by genomewide paired-end sequencing identifies HACE1 as a putative Wilms tumour susceptibility gene. J. Med. Genet. 47, 342-347 (2010).

6. Zhang, L. et al. The E3 ligase HACE1 is a critical chromosome 6q21 tumor suppressor involved in multiple cancers. Nat. Med. 13, 1060-1069 (2007).

7. Diehl, J. A. Cycling to cancer with cyclin D1. Cancer Biol. Ther. 1, 226-231 (2002).

8. Musgrove, E. A., Caldon, C. E., Barraclough, J., Stone, A. \& Sutherland, R. L. Cyclin D as a therapeutic target in cancer. Nat. Rev. Cancer 11, 558-572 (2011).

9. Torrino, S. et al. The E3 ubiquitin-ligase HACE1 catalyzes the ubiquitylation of active Rac1. Dev. Cell 21, 959-965 (2011).

10. Castillo-Lluva, S., Tan, C. T., Daugaard, M., Sorensen, P. H. \& Malliri, A. The tumour suppressor HACE1 controls cell migration by regulating Racl degradation. Oncogene 13, 1735-1742 (2012).

11. Doanes, A. M., Irani, K., Goldschmidt-Clermont, P. J. \& Finkel, T. A requirement for racl in the PDGF-stimulated migration of fibroblasts and vascular smooth cells. Biochem. Mol. Biol. Int. 45, 279-287 (1998).

12. Chou, M. M. \& Blenis, J. The $70 \mathrm{kDa}$ S6 kinase complexes with and is activated by the Rho family G proteins Cdc42 and Rac1. Cell 85, 573-583 (1996).

13. Naumann, M. et al. Activation of activator protein 1 and stress response kinases in epithelial cells colonized by Helicobacter pylori encoding the cag pathogenicity island. J. Biol. Chem. 274, 31655-31662 (1999).

14. Debidda, M., Wang, L., Zang, H., Poli, V. \& Zheng, Y. A role of STAT3 in Rho GTPase-regulated cell migration and proliferation. J. Biol. Chem. 280, 17275-17285 (2005)

15. Cheng, G., Diebold, B. A., Hughes, Y. \& Lambeth, J. D. Nox1-dependent reactive oxygen generation is regulated by Rac1. J. Biol. Chem. 281, $17718-17726$ (2006)

16. Ueyama, T., Geiszt, M. \& Leto, T. L. Involvement of Rac1 in activation of multicomponent Nox1- and Nox3-based NADPH oxidases. Mol. Cell. Biol. 26, 2160-2174 (2006).

17. Yang, T. et al. POSH Localizes activated Racl to control the formation of cytoplasmic dilation of the leading process and neuronal migration. Cell Rep. 12, 2211-1247 (2012).

18. Michaelson, D. et al. Rac1 accumulates in the nucleus during the G2 phase of the cell cycle and promotes cell division. J. Cell. Biol. 181, 485-496 (2008).

19. Oberoi, T. K. et al. IAPs regulate the plasticity of cell migration by directly targeting Rac1 for degradation. EMBO J. 31, 14-28 (2011).

20. Trachootham, D., Alexandre, J. \& Huang, P. Targeting cancer cells by ROSmediated mechanisms: a radical therapeutic approach? Nat. Rev. Drug. Discov. 8, 579-591 (2009).

21. Bedard, K. \& Krause, K. H. The NOX family of ROS-generating NADPH oxidases: physiology and pathophysiology. Physiol. Rev. 87, 245-313 (2007).

22. Chang, H. H. et al. The mechanisms of cytotoxicity of urethane dimethacrylate to Chinese hamster ovary cells. Biomaterials 31, 6917-6925 (2010).
23. Korystov, Y. N., Shaposhnikova, V. V., Korystova, A. F. \& Emel'yanov, M. O Detection of reactive oxygen species induced by radiation in cells using the dichlorofluorescein assay. Radiat. Res. 168, 226-232 (2007).

24. Salas-Vidal, E. et al. Reactive oxygen species participate in the control of mouse embryonic cell death. Exp. Cell. Res. 238, 136-147 (1998)

25. Sorescu, D. et al. Superoxide production and expression of nox family proteins in human atherosclerosis. Circulation 105, 1429-1435 (2002).

26. Soh, N. Recent advances in fluorescent probes for the detection of reactive oxygen species. Anal. Bioanal. Chem. 386, 532-543 (2006).

27. O’Donnell, B. V., Tew, D. G., Jones, O. T. \& England, P. J. Studies on the inhibitory mechanism of iodonium compounds with special reference to neutrophil NADPH oxidase. Biochem. J. 290(Pt 1): 41-49 (1993).

28. Stolk, J., Hiltermann, T. J., Dijkman, J. H. \& Verhoeven, A. J. Characteristics of the inhibition of NADPH oxidase activation in neutrophils by apocynin, a methoxy-substituted catechol. Am. J. Respir. Cell. Mol. Biol. 11, 95-102 (1994).

29. Gianni, D. et al. A novel and specific NADPH oxidase-1 (Nox1) small-molecule inhibitor blocks the formation of functional invadopodia in human colon cancer cells. ACS Chem. Biol. 5, 981-993 (2010).

30. Miyano, K., Ueno, N., Takeya, R. \& Sumimoto, H. Direct involvement of the small GTPase Rac in activation of the superoxide-producing NADPH oxidase Nox1. J. Biol. Chem. 281, 21857-21868 (2006).

31. Juhasz, A. et al. Expression of NADPH oxidase homologues and accessory genes in human cancer cell lines, tumours and adjacent normal tissues. Free Radic. Res. 43, 523-532 (2009).

32. Hordijk, P. L. Regulation of NADPH oxidases: the role of Rac proteins. Circ. Res. 98, 453-462 (2006).

33. Guo, Z., Kozlov, S., Lavin, M. F., Person, M. D. \& Paull, T. T. ATM activation by oxidative stress. Science 330, 517-521 (2010).

34. Raj, L. et al. Selective killing of cancer cells by a small molecule targeting the stress response to ROS. Nature 475, 231-234 (2011).

35. Ciccia, A. \& Elledge, S. J. The DNA damage response: making it safe to play with knives. Mol. Cell 40, 179-204 (2010).

36. Riley, P. A. Free radicals in biology: oxidative stress and the effects of ionizing radiation. Int. J. Radiat. Biol. 65, 27-33 (1994).

37. Kang, M. A., So, E. Y., Simons, A. L., Spitz, D. R. \& Ouchi, T. DNA damage induces reactive oxygen species generation through the H2AX-Nox1/Racl pathway. Cell Death Dis. 3, e249 (2012).

38. Ranjan, P. et al. Redox-dependent expression of cyclin D1 and cell proliferation by Nox1 in mouse lung epithelial cells. Antioxid. Redox. Signal. 8, 1447-1459 (2006).

39. Huttemann, M., Lee, I., Grossman, L. I., Doan, J. W. \& Sanderson, T. H. Phosphorylation of mammalian cytochrome $\mathrm{c}$ and cytochrome $\mathrm{c}$ oxidase in the regulation of cell destiny: respiration, apoptosis, and human disease. Adv. Exp. Med. Biol. 748, 237-264 (2012).

40. Finkel, T. Oxygen radicals and signaling. Curr. Opin. Cell. Biol. 10, 248-253 (1998).

41. DeNicola, G. M. et al. Oncogene-induced Nrf2 transcription promotes ROS detoxification and tumorigenesis. Nature 475, 106-109 (2011).

42. Wertheimer, E. et al. Rac signaling in breast cancer: a tale of GEFs and GAPs. Cell. Signal. 24, 353-362 (2012).

43. Malhotra, D. et al. Global mapping of binding sites for Nrf2 identifies novel targets in cell survival response through ChIP-Seq profiling and network analysis. Nucleic Acids Res. 38, 5718-5734 (2010).

44. Jiang, F., Zhang, Y. \& Dusting, G. J. NADPH oxidase-mediated redox signaling roles in cellular stress response, stress tolerance, and tissue repair. Pharmacol. Rev. 63, 218-242 (2011).

45. Qiu, R. G., Chen, J., Kirn, D., McCormick, F. \& Symons, M. An essential role for Rac in Ras transformation. Nature 374, 457-459 (1995).

46. Irani, K. et al. Mitogenic signaling mediated by oxidants in Ras-transformed fibroblasts. Science 275, 1649-1652 (1997).

47. Gianni, D., Bohl, B., Courtneidge, S. A. \& Bokoch, G. M. The involvement of the tyrosine kinase c-Src in the regulation of reactive oxygen species generation mediated by NADPH oxidase-1. Mol. Biol. Cell 19, 2984-2994 (2008).

48. Ferraro, D. et al. Pro-metastatic signaling by c-Met through RAC-1 and reactive oxygen species (ROS). Oncogene 25, 3689-3698 (2006).

49. Factor, V. M., Kiss, A., Woitach, J. T., Wirth, P. J. \& Thorgeirsson, S. S. Disruption of redox homeostasis in the transforming growth factor-alpha/cmyc transgenic mouse model of accelerated hepatocarcinogenesis. J. Biol. Chem. 273, 15846-15853 (1998).

50. Wondrak, G. T. Redox-directed cancer therapeutics: molecular mechanisms and opportunities. Antioxid. Redox. Signal. 11, 3013-3069 (2009).

51. Sawai, C. M. et al. Therapeutic targeting of the cyclin D3:CDK4/6 complex in T cell leukemia. Cancer Cell 22, 452-465 (2012).

52. Choi, Y. J. et al. The requirement for cyclin d function in tumor maintenance. Cancer Cell 22, 438-451 (2012).

53. Murdoch, C. E., Grieve, D. J., Cave, A. C., Looi, Y. H. \& Shah, A. M. NADPH oxidase and heart failure. Curr. Opin. Pharmacol. 6, 148-153 (2006). 
54. Sorolla, M. A. et al. Proteomic and oxidative stress analysis in human brain samples of Huntington disease. Free. Radic. Biol. Med. 45, 667-678 (2008).

55. Valko, M. et al. Free radicals and antioxidants in normal physiological functions and human disease. Int. J. Biochem. Cell. Biol. 39, 44-84 (2007).

56. Droge, W. Free radicals in the physiological control of cell function. Physiol. Rev. 82, 47-95 (2002).

57. Castillo-Lluva, S. et al. SUMOylation of the GTPase Rac1 is required for optimal cell migration. Nat. Cell. Biol. 12, 1078-1085 (2010).

58. Westerfield, M. The Zebrafish Book. A Guide for the Laboratory Use of Zebrafish (Danio rerio). 4th edn, (University of Oregon Press, 2000).

59. Dobson, J. T. et al. Carboxypeptidase A5 identifies a novel mast cell lineage in the zebrafish providing new insight into mast cell fate determination. Blood 112, 2969-2972 (2008).

60. Niethammer, P., Grabher, C., Look, A. T. \& Mitchison, T. J. A tissue-scale gradient of hydrogen peroxide mediates rapid wound detection in zebrafish. Nature 459, 996-999 (2009).

\section{Acknowledgements}

We thank Joan Mathers for technical assistance. This work was supported in part by funds to PHBS from the British Columbia Cancer Foundation through generous donations from Team Finn and other riders in the Ride to Conquer Cancer, and from the Canadian Institutes of Health Research (CIHR). M.D. was supported by a post-doctoral fellowship from the Danish Cancer Society Scientific Committee. S.T., E.L. and A.M. are supported by funding from the Agence Nationale de la Recherche (ANR-11BSV300401), and a grant from the Association pour la Recherche sur le Cancer (ARC SFI20111203659). A.Mi is supported by funding from Cancer Research UK (grant number C147/A12328) and the Association for International Cancer Research (grant number 12-0037).

\section{Author contributions}

M.D., J.N.B., E.L., A.M., J.M.P. and P.H.B.S. designed the research, M.D., R.N., L.L., B.Ra, L.M., A.J. and A.E.N. performed the experiments. S.C.L., A.Mi, B.R., and A.M. provided useful reagents and helpful discussions, and P.H.B.S., and M.D. wrote the manuscript.

\section{Additional information}

Supplementary Information accompanies this paper at http://www.nature.com/ naturecommunications

Competing financial interests: The authors declare no competing financial interests.

Reprints and permission information is available online at http://npg.nature.com/ reprintsandpermissions/

How to cite this article: Daugaard, M. et al. Hacel controls ROS generation of vertebrate Racl-dependent NADPH oxidase complexes. Nat. Commun. 4:2180 doi: 10.1038/ ncomms3180 (2013)

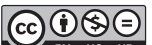

This work is licensed under a Creative Commons AttributionNonCommercial-NoDerivs 3.0 Unported License. To view a copy of this license, visit http://creativecommons.org/licenses/by-nc-nd/3.0/ 